\title{
Aharonov-Bohm physics with spin. I. Geometric phases in one-dimensional ballistic rings
}

\author{
Martina Hentschel \\ Department of Physics, Duke University, Box 90305, Durham, North Carolina 27708-0305, USA \\ Henning Schomerus \\ Max-Planck-Institut für Physik komplexer Systeme, Nöthnitzer Straße 38, 01187 Dresden, Germany \\ Diego Frustaglia \\ Institut für Theoretische Festkörperphysik, Universität Karlsruhe, 76128 Karlsruhe, Germany
}

Klaus Richter

Institut für Theoretische Physik, Universität Regensburg, 93040 Regensburg, Germany

(Received 18 September 2003; published 27 April 2004)

\begin{abstract}
We analytically calculate the spin-dependent electronic conductance through a one-dimensional ballistic ring in the presence of an inhomogeneous magnetic field and identify signatures of geometric and Berry phases in the general nonadiabatic situation. For an in-plane magnetic field, we rigorously prove the spin-flip effect presented by Frustaglia et al. [Phys. Rev. Lett. 87, 256602 (2001)], which allows us to control and switch the polarization of outgoing electrons by means of an Aharonov-Bohm flux, and derive analytical expressions for the energy-averaged magnetoconductance. Our results support numerical calculations for two-dimensional ballistic rings presented in the second paper [Frustaglia et al., following paper, Phys. Rev. B 69, 155327 (2004)] of this series.
\end{abstract}

DOI: 10.1103/PhysRevB.69.155326

PACS number(s): 73.23.-b, 03.65.Vf, 05.30.Fk, 72.25.-b

\section{INTRODUCTION}

The Aharonov-Bohm $(\mathrm{AB})$ effect $^{2}$ represents a genuine interference phenomenon at the heart of mesoscopic quantum physics. ${ }^{3}$ It has allowed us to probe the coherence of wave functions extending over ring conductors of micron scales by monitoring the magnetoconductance as a function of a magnetic flux threaded through the ring. To date the AB effect is being used as a tool to investigate phase coherence and dephasing mechanisms in nanostructures. In common $\mathrm{AB}$ setups composed of metal or semiconductor rings subject to uniform flux-generating external magnetic fields, the relevant physics is governed by (interference of) the orbital part of the electron wave function, while the spin degree of freedom can usually be neglected. More recently, the role of the electron spin as a means, besides charge, to control a current or to store information has received much attention in the context of spintronics. ${ }^{4}$ Electrons with spin experience quantum phases beyond the charge-based $\mathrm{AB}$ phase. The subject of this paper is the study of the resulting complex interference phenomena on the theoretical side.

The adiabatic Berry phase ${ }^{5}$ and nonadiabatic AharonovAnandan geometric phases ${ }^{6,7}$ are key aspects of electronic transport in inhomogeneous magnetic fields. Just as other phases (such as scattering phases in the Coulomb blockade regime $^{8,9}$ and of Kondo ${ }^{10-12}$ or Fano resonances ${ }^{13,14}$ ) they can be probed by interference experiments, which most easily are carried out in the AB ring geometry. Since the spin degree of freedom becomes a dynamical quantity in inhomogeneous magnetic fields, its generation of, and interplay with, geometric and Berry phases deserves detailed investigation. ${ }^{15-21}$
This is the first paper of a two-part series on spindependent electronic transport through rings in the presence of inhomogeneous magnetic fields. In this first part we address ballistic spin-dependent transport through circular rings in rotationally symmetric magnetic fields that occur in realizations of inhomogeneous fields by a central micromagnet ${ }^{22}$ or by a current through a wire piercing the ring; ${ }^{23}$ see Fig. 1. For narrow confinement the orbital transport channels decouple, and it suffices to investigate the reduced strictly onedimensional (1D) problem for each channel, which, as we will show, can be solved exactly for all strengths of the magnetic field.

The constraint of rotational invariance is relaxed in the second part $^{1}$ of this series (also referred to as paper II in the following), where three of us describe a general numerical Green-function approach to the spin-dependent magnetocon- (a)

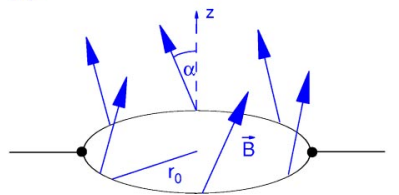

(b)

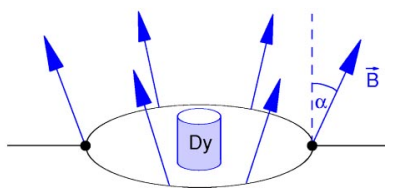

FIG. 1. Magnetic-field texture for (a) a wirelike and (b) a crownlike magnetic field. The angle $\alpha$ is defined as the angle of the magnetic field with respect to the $z$ axis. A possible way for creating a wirelike magnetic field in experiments is by means of a central current lead, while a crownlike field can be obtained by placing a micromagnet (like Dysprosium) into the center of the ring. Besides the homogeneous magnetic field in $z$ direction, a circular (tangential) or a radial magnetic component is then present, respectively. Both field textures are rotationally invariant about the $z$ axis. 
ductance, which works for arbitrary geometry of the system and texture of the magnetic field, and also in the presence of disorder. This approach is applied there to two-dimensional rings, and for a rotationally symmetric in-plane magnetic field a spin-flip effect is found, which allows us to control and switch the polarization of ballistic electrons by a small Aharonov-Bohm flux through the ring (for a short exposition of this effect see Ref. 24). In the present paper (paper I) we provide a strict analytical proof of the spin-flip effect, and derive analytical results that support our findings for the twodimensional (2D) rings presented in paper II.

The outline of this paper is as follows. In Sec. II we introduce our model of a 1D ring subject to an inhomogeneous magnetic field and present the general solution of the Schrödinger equation. Section III is devoted to the computation of the magnetoconductance within a transfer-matrix approach. Generalizing the spin-independent transport discussed in Sec. III A, spin dependence is taken into account in Sec. III B. Results are presented in Sec. IV, where in particular we consider a ring with a central micromagnet and discuss the appearance of geometric phases in the magnetoconductance. Section V deals with the special case of an in-plane magnetic field. We analytically prove the spin-flip effect in Sec. V A. In Secs. V B and V C we discuss the transition from the nonadiabatic to the adiabatic situation in terms of the energy-averaged conductance, and derive simple analytical expressions that explain the observed features. We finish with a discussion of our results in Sec. VI.

\section{ONE-DIMENSIONAL RING IN AN INHOMOGENEOUS MAGNETIC FIELD}

We consider the spin-dependent coherent electronic transport through a circular ring of radius $r_{0}$ within a layer of a two-dimensional electron gas (2DEG), exposed to an inhomogeneous magnetic field $\vec{B}(\vec{r})$. The transport is assumed to be ballistic, i.e., the ring contains no impurities, and electronelectron interactions are ignored. The charge carriers in the 2DEG are characterized by their electric charge $-e<0$, effective mass $m^{*}$, and magnetic moment $\mu=\frac{1}{2} g^{*} \mu_{B}$, where $g^{*}$ is the effective gyromagnetic ratio and $\mu_{B}=e \hbar /\left(2 m_{0} c\right)$ is Bohr's magneton with $m_{0}$ being the bare electron mass. For free electrons in vacuum, the gyromagnetic ratio $g^{*}$ $=g$ is $\approx 2$. However, for electronlike quasiparticles in semiconductor heterostructures, considerable deviations from this value occur depending on the material. We set $\hbar=1$ throughout the rest of the paper and introduce scaled parameters for the magnetic moment, $\tilde{\mu}=2 m^{*} r_{0}^{2} \mu$, and the Fermi energy in the ring, $\widetilde{E}_{F}=2 m^{*} r_{0}^{2} E_{F}=\left(k_{F} r_{0}\right)^{2}$.

The magnetic field $\vec{B}(\vec{r})$ couples to both the spin and the orbital degrees of freedom. Spin-orbit interaction is assumed to be small, and will be neglected. The effect of Rashba spin-orbit coupling ${ }^{25}$ is an additional in-plane magnetic-field component which depends on the Fermi energy. The corresponding term is similar to the Aharonov-Casher phase term considered in Ref. 26, and not investigated here. The Hamiltonian within the confined region then reads

$$
H=\frac{1}{2 m^{*}}\left(\vec{p}+\frac{e}{c} \vec{A}_{\mathrm{em}}(\vec{r})\right)^{2}+\mu \vec{\sigma} \cdot \vec{B}(\vec{r}),
$$

with $\vec{A}_{\mathrm{em}}(\vec{r})$ the vector potential, $\vec{B}(\vec{r})=\vec{\nabla} \times \vec{A}_{\mathrm{em}}(\vec{r})$, and $\vec{\sigma}$ the vector of Pauli spin matrices. The first term in the Hamiltonian (1) describes the kinetic energy and involves the generalized momentum $\vec{\Pi}=\vec{p}+(e / c) \vec{A}_{\mathrm{em}}(\vec{r})$. The second term $\mu \vec{\sigma} \cdot \vec{B}(\vec{r})$ corresponds to the Zeeman coupling of the electron spin $\vec{\sigma}$ to the magnetic field $\vec{B}(\vec{r})$.

We place the ring into the $x y$ plane and decompose the magnetic field in cylindrical coordinates $\vec{B}=B_{r} \vec{e}_{r}+B_{\varphi} \vec{e}_{\varphi}$ $+B_{z} \vec{e}_{z}$, where the polar angle $\varphi$ parametrizes the position along the ring. We investigate the two magnetic-field textures shown in Fig. 1, which will be distinguished by the parameter

$$
\varphi_{t}= \begin{cases}\frac{\pi}{2} & \text { for a wirelike magnetic field } \\ 0 & \text { for a crownlike magnetic field }\end{cases}
$$

The textures are further characterized by the tilt angle $\alpha$ of the magnetic field with respect to the perpendicular $z$ axis. The total magnetic flux through the ring is denoted by $\phi^{\mathrm{AB}}$ $=\phi_{z}^{\mathrm{AB}}+\phi_{\mathrm{ext}}^{\mathrm{AB}}$, which includes the contribution $\phi_{z}^{\mathrm{AB}}=\pi r_{0}^{2} B_{z}$ generated by the magnetic field in the ring itself, as well as an extra magnetic flux $\phi_{\mathrm{ext}}^{\mathrm{AB}}$ that can be generated by an homogeneous magnetic field perpendicular to the ring plane, or a solenoid piercing the ring. The magnetic flux quantum is denoted by $\phi_{0}=e / 2 \pi$, and the total strength of the magnetic field by $B=|\vec{B}|$.

The eigenstates $\left|\Psi_{n}^{\uparrow, \downarrow}\right\rangle$ of the Hamiltonian (1) can be found analytically following Refs. 16 and 26 . They can be decomposed into an orbital part $\psi_{n}^{\uparrow, \downarrow}(\varphi)$ and a spin part $\left|s_{n}^{\uparrow, \downarrow}(\varphi)\right\rangle$, i.e., $\left\langle\varphi \mid \Psi_{n}^{\uparrow, \downarrow}\right\rangle=\psi_{n}^{\uparrow, \downarrow}(\varphi)\left|s_{n}^{\uparrow, \downarrow}(\varphi)\right\rangle$.

The orbital functions $\psi_{n}^{\uparrow, \downarrow}(\varphi)$ of the Hamiltonian (1) are plane waves $e^{i n \varphi}$. Since later we will consider the effect of the Aharonov-Bohm flux $\phi^{\mathrm{AB}}$ and the transport through open rings, we will not require the orbital quantum number $n$ to be an integer, which equips us with a basis for scattering states at arbitrary energy. This still leaves us with the problem to find for a given energy the correct values of $n$, as well as the spin directions.

The spin part will be written in the $S_{\alpha}$ basis of spin eigenstates $|\uparrow(\varphi)\rangle,|\downarrow(\varphi)\rangle$ of the Zeeman term, which in turn read $\left(\varphi^{\prime} \equiv \varphi+\varphi_{t}\right)$

$$
\begin{aligned}
& \left(\begin{array}{c}
\langle\Uparrow \mid \uparrow(\varphi)\rangle \\
\langle\Downarrow \mid \uparrow(\varphi)\rangle
\end{array}\right)=\left(\begin{array}{c}
\cos \frac{\alpha}{2} \\
e^{i \varphi^{\prime}} \sin \frac{\alpha}{2}
\end{array}\right), \\
& \left(\begin{array}{c}
\langle\Uparrow \mid \downarrow(\varphi)\rangle \\
\langle\Downarrow \mid \downarrow(\varphi)\rangle
\end{array}\right)=\left(\begin{array}{c}
\sin \frac{\alpha}{2} \\
-e^{i \varphi^{\prime}} \cos \frac{\alpha}{2}
\end{array}\right)
\end{aligned}
$$


in the $S_{z}$ basis $|\Uparrow\rangle,|\Downarrow\rangle$ of the spin-up and spin-down eigenstates of $\sigma_{z}$. The total eigenstates of the Zeeman term (including the orbital part) will be denoted by ||$\left.\left.\uparrow_{n}\right\rangle\right\rangle$ and ||$\left.\left.\downarrow_{n}\right\rangle\right\rangle$, with $\left.\left\langle\varphi|| \uparrow_{n}\right\rangle\right\rangle=\exp (\operatorname{in} \phi)|\uparrow(\varphi)\rangle \quad$ and $\left.\left\langle\varphi|| \downarrow_{n}\right\rangle\right\rangle$ $=\exp (\operatorname{in} \phi)|\downarrow(\varphi)\rangle$.

The spin quantum number $\sigma=\uparrow, \downarrow$ of $\left|\Psi_{n}^{\sigma}\right\rangle$ indicates whether the state aligns parallel $(\sigma=\uparrow)$ or antiparallel $(\sigma$ $=\downarrow$ ) with the magnetic field when its strength is increased. One then enters the adiabatic regime, in which the eigenstates $\left.\left.\left|\Psi_{n}^{\sigma}\right\rangle \sim \| \sigma_{n}\right\rangle\right\rangle$, and the spins never switch their direction with respect to the magnetic field during the transport through the ring. This requires a large ratio

$$
Q=\frac{\omega_{L}}{\omega_{\text {orb }}}=\frac{\tilde{\mu} B}{k_{F} r_{0}}
$$

of the spin precession (Larmor) frequency

$$
\omega_{L}=\frac{g * e B}{2 m_{0}}=\frac{\tilde{\mu} B}{r_{0}^{2} m^{*}}
$$

and the orbital frequency

$$
\omega_{\mathrm{orb}}=\frac{v_{F}}{r_{0}}=\frac{k_{F} r_{0}}{r_{0}^{2} m^{*}} .
$$

In the adiabatic regime the two subspaces spanned by the states ||$\left.\left.\uparrow_{n}\right\rangle\right\rangle$ and ||$\left.\left.\downarrow_{n}\right\rangle\right\rangle$ are completely decoupled. In order to solve the problem under nonadiabatic conditions, it is useful to decompose the Hamiltonian (1) into two parts, $H=H_{0}$ $+H_{1}$, where the adiabatic part $H_{0}$ contains no transitions between the ||$\left.\left.\uparrow_{n}\right\rangle\right\rangle$ and $\left.\left.\| \downarrow_{n}\right\rangle\right\rangle$ subspaces, whereas the nonadiabatic part $H_{1}$ exclusively describes such transitions. To perform this decomposition, one can apply the concept of a geometric vector potential $A_{g}$ introduced by Aharonov and Anandan in Ref. 6. The result reads ${ }^{16,17}$

$$
\begin{gathered}
H_{0}=\frac{1}{2 m^{*}}\left[\left(\Pi-A_{g}\right)^{2}+A_{g}^{2}\right]+\mu \vec{\sigma} \cdot \vec{B}, \\
H_{1}=\frac{1}{2 m^{*}}\left[\left(\Pi-A_{g}\right) A_{g}+A_{g}\left(\Pi-A_{g}\right)\right],
\end{gathered}
$$

with the generalized momentum operator

$$
\Pi=-\frac{i}{r_{0}} \frac{d}{d \varphi}+e A_{\mathrm{em}}^{\varphi}
$$

and the geometric vector potential

$$
A_{g}=\frac{\sin \alpha}{2 r_{0}}\left(\begin{array}{cc}
-\sin \alpha & \cos \alpha e^{-i \varphi^{\prime}} \\
\cos \alpha e^{i \varphi^{\prime}} & \sin \alpha
\end{array}\right)
$$

The geometric vector potential $A_{g}$ causes the nonadiabatic geometric $^{6,7}$ and adiabatic Berry phases. ${ }^{5}$ Note that only the direction $\alpha$ of the magnetic field at the position of the ring enters the expression for $A_{g}$ explicitly, and that $\alpha$ changes under variation of an external homogeneous magnetic field.
However, $A_{g}$ is not affected by solenoid-generated external Aharonov-Bohm fluxes [that, of course, contribute to $\phi_{\mathrm{ext}}^{\mathrm{AB}}$ and $\phi^{\mathrm{AB}}$ and alter the problem via, e.g., Eq. (11)].

The exact eigenstates of the Hamiltonian (1) now can be found in the $S_{\alpha}$ basis (3),

$$
\left\langle\varphi \mid \Psi_{n}^{\uparrow, \downarrow}\right\rangle=\psi_{n}(\varphi)\left[C_{1, n}^{\uparrow, \downarrow}|\uparrow(\varphi)\rangle+C_{2, n}^{\uparrow, \downarrow}|\downarrow(\varphi)\rangle\right],
$$

where the coefficients $C_{1, n}^{\uparrow, \downarrow}$ and $C_{2, n}^{\uparrow, \downarrow}$ are obtained from the eigenvalue equation

$$
\left(\begin{array}{ll}
H_{0}^{\uparrow \uparrow} & H_{1}^{\uparrow \downarrow} \\
H_{1}^{\downarrow \uparrow} & H_{0}^{\downarrow \downarrow}
\end{array}\right)\left(\begin{array}{c}
C_{1, n}^{\uparrow, \downarrow} \\
C_{2, n}^{\uparrow, \downarrow}
\end{array}\right) \psi_{n}(\varphi)=E_{n}\left(\begin{array}{c}
C_{1, n}^{\uparrow, \downarrow} \\
C_{2, n}^{\uparrow, \downarrow}
\end{array}\right) \psi_{n}(\varphi)
$$

with $H_{l}^{\sigma \sigma^{\prime}}=\left\langle\sigma(\varphi)\left|H_{l}\right| \sigma^{\prime}(\varphi)\right\rangle$. The diagonal elements describe the adiabatic part of the problem, whereas the nondiagonal entries contain the nonadiabatic (spin-flip) processes that vanish in the adiabatic regime.

Straightforward algebra yields that $\left(\begin{array}{c}C_{2, n}^{\uparrow} \\ C_{1, n}^{\uparrow}\end{array}\right)$ and $\left(\begin{array}{c}C_{2, n}^{\downarrow} \\ C_{1, n}^{\downarrow}\end{array}\right)$ are the two eigenvectors of the matrix ${ }^{16,17}$

$$
\left(\begin{array}{cc}
n^{\prime 2}+\eta \sin ^{2} \frac{\alpha}{2}+\tilde{\mu} B & -\frac{\eta}{2} \sin \alpha \\
-\frac{\eta}{2} \sin \alpha & n^{\prime 2}+\eta \cos ^{2} \frac{\alpha}{2}-\tilde{\mu} B
\end{array}\right)
$$

with $\eta=2 n^{\prime}+1$ and

$$
n^{\prime}=n+\phi^{\mathrm{AB}} / \phi_{0},
$$

which can be identified as the quantum number of the generalized momentum $\Pi$. For given Fermi energy $\widetilde{E}_{F}$, the four solutions $n_{\rho}^{\prime \sigma}$ of the equation

$$
\begin{aligned}
0= & n^{\prime 4}+2 n^{\prime 3}+\left(1-2 \widetilde{E}_{F}\right) n^{\prime 2}-2\left(\widetilde{E}_{F}-\tilde{\mu} B \cos \alpha\right) n^{\prime}+\widetilde{E}_{F}^{2} \\
& -\widetilde{E}_{F}+\tilde{\mu} B \cos \alpha-(\tilde{\mu} B)^{2}
\end{aligned}
$$

can be associated to the four combinations of counterclockwise $(\rho=+)$ and clockwise $(\rho=-)$ motion with spin up ( $\sigma=\uparrow$ ) or down $(\sigma=\downarrow)$. The propagation sense is distinguished according to the criterion $n_{+}^{\prime \sigma}+1 / 2>0, n_{-}^{\prime \sigma}+1 / 2$ $<0$. In the $S_{z}$ basis, the corresponding spin eigenstates are given by

$$
\begin{aligned}
& \left(\begin{array}{c}
\left\langle\Uparrow \mid s_{n_{\rho}}^{\uparrow}\right\rangle \\
\left\langle\Downarrow \mid s_{n_{\rho}}^{\uparrow}\right\rangle
\end{array}\right)=\left(\begin{array}{c}
\cos \frac{\gamma_{\rho}^{\uparrow}}{2} \\
\rho e^{i \varphi^{\prime}} \sin \frac{\gamma_{\rho}^{\uparrow}}{2}
\end{array}\right), \\
& \left(\begin{array}{c}
\left\langle\Uparrow \mid s_{n_{\rho}}^{\downarrow}\right\rangle \\
\left\langle\downarrow \mid s_{n_{\rho}}^{\downarrow}\right\rangle
\end{array}\right)=\left(\begin{array}{c}
\gamma_{\rho}^{\downarrow} \\
\sin \frac{1}{2} \\
-\rho e^{i \varphi^{\prime}} \cos \frac{\gamma_{\rho}^{\downarrow}}{2}
\end{array}\right),
\end{aligned}
$$

with the angles 


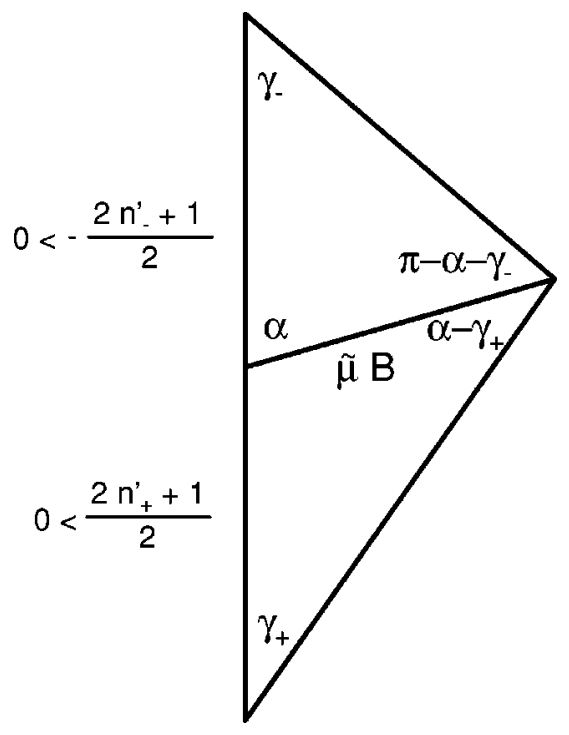

FIG. 2. Geometric interpretation of the angles $\gamma$ depending on the ratio of kinetic $\left(n^{\prime}+1 / 2\right)$ and magnetic $(\tilde{\mu} B)$ energies, and the (fixed) angle $\alpha$. For $\alpha>\pi / 2$, the definitions of $n_{+}^{\prime}$ and $n_{-}^{\prime}$ have to be interchanged.

$$
\cot \gamma_{\rho}^{\sigma}=\rho\left[\cot \alpha+\frac{\left(2 n_{\rho}^{\prime \sigma}+1\right)}{2} \frac{1}{\tilde{\mu} B \sin \alpha}\right] .
$$

The spin states (13) are of the form of Eq. (3) with $\alpha$ replaced by $\gamma_{\rho}^{\sigma}$. These angles have an illustrative geometric interpretation shown in Fig. 2. In the adiabatic limit, $\tilde{\mu} B$ $\gg\left|n^{\prime}\right|$, we find $\gamma_{+}^{\sigma}=\alpha$ and $\gamma_{-}^{\sigma}=\pi-\alpha$, whereas in the diabatic limit $\gamma_{\rho}^{\sigma}=0$.

This substitution rule $\alpha \rightarrow \gamma_{\rho}^{\sigma}$ also applies to the generalization of the adiabatic Berry phase ${ }^{5}$ to nonadiabatic geometric Aharonov-Anandan phases: ${ }^{6,7}$ Here, $\gamma_{\rho}^{\sigma}$ replaces $\alpha$ as the solid angle enclosed during one round trip in parameter space. For the states $\left|\Psi_{n_{+}}^{\sigma}\right\rangle$, where $0<\gamma_{+}^{\sigma}<\alpha$, this nicely follows the intuitive interpretation that in the nonadiabatic case the magnetic field is not strong enough to force the spin into the direction of $\alpha$, but only to a smaller angle $\gamma_{+}^{\sigma}$. In this sense $\left|\left(\gamma_{+}^{\sigma}-\alpha\right) / \alpha\right|$ can be taken as a qualitative measure for the deviation from the adiabatic case: Starting with $\gamma_{+}^{\sigma}$ $=\alpha$ in the adiabatic situation, this quantity increases from zero to eventually reach 1 in the diabatic limit when $\gamma_{+}^{\sigma}$ $=0$. Indeed, for the special case of an in-plane magnetic field (Sec. V) we will see that the adiabaticity parameter $Q$ defined in Eq. (4) is directly related to the values of $\gamma_{+}^{\sigma}$, cf. Eq. (58).

\section{MAGNETOCONDUCTANCE}

\section{A. Spin-independent transport}

We now investigate the magnetoconductance through a 1D ring within a transfer-matrix approach. For phasecoherent transport, the conductance $G=\left(e^{2} / h\right) \Sigma_{\sigma, \sigma^{\prime}} T_{\sigma \sigma^{\prime}}$ is given by the transmission probabilities $T_{\sigma \sigma^{\prime}}$ between the spin channels $\sigma, \sigma^{\prime}=\uparrow, \downarrow$ in each lead. We will work with

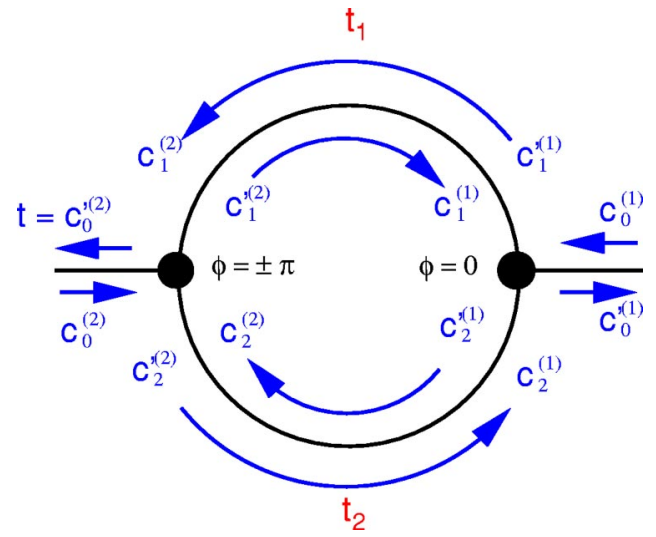

FIG. 3. Definition of the transmission and reflection amplitudes in a $1 \mathrm{D}$ ring coupled to current leads.

the dimensionless conductance $T=\Sigma_{\sigma, \sigma^{\prime}} T_{\sigma \sigma^{\prime}}$. In the adiabatic case, the transport is spin independent, and it suffices to study one spin species. ${ }^{27}$ We first review this case and then generalize it to the nonadiabatic situation, partially following Ref. 26.

We describe each of the two identical junctions $(i=1,2)$ between the leads and the $1 \mathrm{D}$ ring by a $3 \times 3$ scattering matrix $S$, which relates, by way of

$$
\mathbf{c}^{\prime(i)}=S \mathbf{c}^{(i)},
$$

the coefficients $\mathbf{c}^{(i)}=\left(c_{0}^{(i)}, c_{1}^{(i)}, c_{2}^{(i)}\right)$ of incoming scattering states to the coefficients $\mathbf{c}^{\prime(i)}=\left(c_{0}^{\prime(i)}, c_{1}^{\prime(i)}, c_{2}^{\prime(i)}\right)$ of outgoing scattering states (cf. Fig. 3). Here the index 0 is assigned to the coefficients in the external leads, the index 1 denotes the upper arm of the ring, and the index 2 denotes the lower arm of the ring. The scattering states are assumed to be orthogonal and normalized to carry unit particle flux. Current conservation and time-reversal symmetry at each junction imply that $S$ is unitary and symmetric, and spatial symmetry leaves only one free parameter $\varepsilon$ (up to phase factors) to characterize the coupling strength between the leads and the ring: A wave is transmitted from the external leads into each of the two branches of the ring with equal probability $\varepsilon$, whereas reflection occurs with probability $1-2 \varepsilon$. In particular, for $\varepsilon=0$, all particles are reflected so that there is no coupling into the (then isolated) ring.

We write $S$ in the conventional form ${ }^{27}$ as

$$
S=\left(\begin{array}{ccc}
-(a+b) & \sqrt{\varepsilon} & \sqrt{\varepsilon} \\
\sqrt{\varepsilon} & a & b \\
\sqrt{\varepsilon} & b & a
\end{array}\right),
$$

where

$$
a=\frac{1}{2}(\sqrt{1-2 \varepsilon}-1), \quad b=a+1 .
$$

Before we turn to the case with spin where the amplitudes $\mathbf{c}^{(i)}, \mathbf{c}^{\prime(i)}$ have spinor character, let us summarize the result for the case without spin from Ref. 27. In this case we can work with the orbital part alone, and the propagation velocities in all scattering states at a given energy are equal because there is no Zeeman energy, which simplifies the nor- 
malization to unit particle flux. Assuming $c_{0}^{(1)}=1, c_{0}^{(2)}=0$, the dimensionless conductance $T=|t|^{2}$ is obtained from

$$
t=c_{0}^{\prime(2)}=-\frac{\varepsilon}{b^{2}}(1,1) t_{1} P\left(\begin{array}{c}
1 \\
-1
\end{array}\right) .
$$

with

$$
\begin{gathered}
P=\left(S_{\mathrm{lr}} t_{2} S_{\mathrm{lr}} t_{1}-1_{2 \times 2}\right)^{-1}, \\
S_{\mathrm{lr}}=\frac{1}{b}\left(\begin{array}{cc}
a+b & a \\
-a & 1
\end{array}\right) .
\end{gathered}
$$

Here, $S_{1 \mathrm{l}}$ relates the amplitudes in the two arms of the ring across the junctions, whereas the transfer matrices $t_{1}, t_{2}$ relate the amplitudes within each arm of the ring (see Fig. 3),

$$
\begin{gathered}
\left(\begin{array}{c}
c_{2}^{\prime(2)} \\
c_{2}^{(2)}
\end{array}\right)=S_{\operatorname{lr}}\left(\begin{array}{c}
c_{1}^{(2)} \\
c_{1}^{\prime(2)}
\end{array}\right), \\
\left(\begin{array}{c}
c_{1}^{(2)} \\
c_{1}^{\prime(2)}
\end{array}\right)=t_{1}\left(\begin{array}{c}
c_{1}^{\prime(1)} \\
c_{1}^{(1)}
\end{array}\right), \quad\left(\begin{array}{c}
c_{2}^{(1)} \\
c_{2}^{\prime(1)}
\end{array}\right)=t_{2}\left(\begin{array}{c}
c_{2}^{\prime(2)} \\
c_{2}^{(2)}
\end{array}\right) .
\end{gathered}
$$

For ballistic transport and symmetric arms, the transfer matrices

$$
t_{1}=t_{2}=e^{-i \theta_{\mathrm{AB}}}\left(\begin{array}{cc}
e^{i \theta_{d}} & 0 \\
0 & e^{-i \theta_{d}}
\end{array}\right)
$$

comprise two phase factors each, namely, the dynamic phase $\theta_{d}=\pi k_{F} r_{0}$ and an Aharonov-Bohm phase $\theta_{\mathrm{AB}}=\pi \phi^{\mathrm{AB}} / \phi_{0}$ arising from the magnetic flux through the ring. For fixed Fermi energy, the dimensionless conductance $T\left(\phi^{\mathrm{AB}}\right)$ shows characteristic Aharonov-Bohm fluctuations. ${ }^{27}$ The energyaveraged dimensionless conductance $\left(\phi^{\mathrm{AB}}=0\right)$ depends on the coupling parameter $\varepsilon$ as

$$
\langle T(\varepsilon)\rangle=\frac{\varepsilon}{1-\varepsilon}
$$

These results for spinless electrons can be directly carried over to electrons with spin in the adiabatic regime, when one corrects the Aharonov-Bohm phase $\theta_{\mathrm{AB}}$ by the geometric phase $^{20}$

$$
\Gamma^{\uparrow(\downarrow)}=\pi[1+(-) \cos \alpha],
$$

following the replacement rule

$$
\theta_{\mathrm{AB}}=\frac{\pi}{\phi_{0}} \phi^{\mathrm{AB}} \rightarrow \theta_{\mathrm{AB}}^{\sigma}=\frac{\pi}{\phi_{0}}\left(\phi^{\mathrm{AB}}-\phi_{0} \frac{\Gamma^{\sigma}}{2 \pi}\right),
$$

and accounts for the Zeeman interaction energy in the dynamical phase,

$$
\theta_{d}=\pi \widetilde{E}_{F}^{1 / 2} \rightarrow \theta_{d}^{\uparrow(\downarrow)}=\pi \sqrt{\widetilde{E}_{F}+(-) \tilde{\mu} B} .
$$

The splitting of $\theta_{d}^{\uparrow(\downarrow)}$ results in interference and beating of the amplitudes of the two electron species due to their slightly different oscillation frequencies, which destroys the $\phi_{0}$ periodicity of the Aharonov-Bohm effect (see also the discussion of Fig. 5).

\section{B. Spin-dependent transport}

In spin-dependent transport, transitions between the two spin channels $|\Uparrow\rangle,|\Downarrow\rangle$ are possible, and the generalization of spin-independent transport requires two changes. First, the wave functions acquire a spin dependence at each spatial position, cf. Sec. II, and all transport equations have to be formulated in the resulting product space (orbital motion $\otimes$ spin state). This is explained in Sec. III B 1. Second, at given total energy the electronic velocities $v_{\rho}^{\Uparrow, \Downarrow}$ depend on the spin and propagation direction, because the Zeeman energy is state dependent, and also the spin states (13) are not orthogonal, as already has been noted in Ref. 26. Here we find it necessary to depart from the derivation of transfer coefficients in Ref. 26, which resulted in nonunitary amplituderelating transfer matrices. This would lead to a total dimensionless conductance $T$ that can take values above two, in contradiction to particle number conservation. If we wish to couple to the leads by the conventional unitary $S$ matrix (16), we hence have to work with suitable flux-normalized, orthogonal scattering states. This is done in Sec. III B 2.

\section{Formalism}

The spin degree of freedom is incorporated formally by upgrading the coefficients c to spinors. Accordingly, they now consist of two components $\mathbf{c}^{\Uparrow}$, $\mathbf{c}^{\Downarrow}$, where $\Uparrow(\Downarrow)$ denotes the spin state in $z$ direction. The transfer matrices $t_{1}, t_{2}$ are now $4 \times 4$ matrices, and the transmission amplitude $t$ in Eq. (17) is the $2 \times 2$ matrix,

$$
t=\left(\begin{array}{cc}
t_{\Uparrow \Uparrow} & t_{\Uparrow \downarrow} \\
t_{\Downarrow \Uparrow} & t_{\Downarrow \Downarrow}
\end{array}\right) .
$$

The four entries of $t$ measure the transmission amplitudes between all possible combinations of the spin states in the $S_{z}$ basis.

For unpolarized incident electrons, the total dimensionless conductance $T$ is given by

$$
T=\sum_{\sigma, \sigma^{\prime}=\Uparrow, \Downarrow}\left|t_{\sigma \sigma^{\prime}}\right|^{2}=T_{\Uparrow \Uparrow}+T_{\Uparrow \Downarrow}+T_{\Downarrow \Uparrow}+T_{\Downarrow \Downarrow} .
$$

Equation (17) for the transmitted amplitude is replaced by

$$
t=-\varepsilon m_{1} t_{1} P m_{2}
$$

with

$$
\begin{aligned}
& P=\left[\left(S_{\mathrm{lr}} \otimes \sigma_{0}\right) t_{2}\left(S_{\mathrm{lr}} \otimes \sigma_{0}\right) t_{1}-1_{2 \times 2} \otimes \sigma_{0}\right]^{-1}, \\
& m_{1}=\frac{1}{b}\left([1,1] \otimes \sigma_{0}\right), \quad m_{2}=\frac{1}{b}\left(\left[\begin{array}{c}
1 \\
-1
\end{array}\right] \otimes \sigma_{0}\right) .
\end{aligned}
$$

Here $\sigma_{0}$ is the $2 \times 2$ unit matrix in spin space. The fluxconserving transfer matrix $t_{1}$ in the upper arm and its counterpart $t_{2}$ in the lower arm are derived in the Sec. III B 2. 
For perfectly symmetric rings we can derive a convenient simplified version of Eq. (26). To this end we introduce the matrix

$$
m_{3}=\frac{1}{b}\left(\begin{array}{cccc}
a+b & 0 & a & 0 \\
0 & a+b & 0 & a \\
-a & 0 & 1 & 0 \\
0 & -a & 0 & 1
\end{array}\right)=N_{1} N_{2}
$$

with the decomposition

$$
\begin{gathered}
N_{1}=\frac{1}{\sqrt{2 b}}\left(\begin{array}{cccc}
b+a & 0 & -1 & 0 \\
0 & b+a & 0 & -1 \\
1 & 0 & 1 & 0 \\
0 & 1 & 0 & 1
\end{array}\right), \\
N_{2}=\frac{1}{\sqrt{2 b}}\left(\begin{array}{cccc}
1 & 0 & 1 & 0 \\
0 & 1 & 0 & 1 \\
-(b+a) & 0 & 1 & 0 \\
0 & -(b+a) & 0 & 1
\end{array}\right) .
\end{gathered}
$$

This allows us to rewrite Eq. (26) in the symmetric form

$$
t=-\varepsilon m_{1} N_{2}^{-1}\left[N_{2} t_{2} N_{1}-N_{1}^{-1} t_{1}^{-1} N_{2}^{-1}\right]^{-1} N_{1}^{-1} m_{2} .
$$

If we furthermore introduce

$$
P^{\prime}=\left[N_{2} t_{2} N_{1}-N_{1}^{-1} t_{1}^{-1} N_{2}^{-1}\right]^{-1},
$$

we see from the previous equation that the term to the left and right of $P^{\prime}$ projects out just the upper right $2 \times 2$ matrix of $P^{\prime}$,

$$
t=-\varepsilon \frac{2}{b}\left(\begin{array}{ll}
P_{13}^{\prime} & P_{14}^{\prime} \\
P_{23}^{\prime} & P_{24}^{\prime}
\end{array}\right),
$$

which simplifies the calculation of the dimensionless conductance from Eqs. (24) and (25).

\section{Computation of the transfer matrices}

We now turn to the computation of the flux-normalized transfer matrices $t_{1}, t_{2}$, taking care for the state dependence of the propagation velocities $v_{\rho}^{\Uparrow(\Downarrow)}$ and for the nonorthogonality of the spin states (13).

In analogy to the spinless case, we first introduce transfer matrices $\tilde{t}_{1}, \tilde{t}_{2}$ that relate the amplitudes rather than the fluxes. With the velocity matrix

$$
v=\operatorname{diag}\left(v_{+}^{\Uparrow}, v_{+}^{\Downarrow}, v_{-}^{\Uparrow}, v_{-}^{\Downarrow}\right)
$$

the relation between $\tilde{t}_{1}, \tilde{t}_{2}$ and $t_{1}, t_{2}$ is given by

$$
t_{1}=v^{1 / 2} \tilde{t}_{1} v^{-1 / 2}, \quad t_{2}=v^{1 / 2} \tilde{t}_{2} v^{-1 / 2} .
$$

Following the wave-matching procedure for ballistic transport in Ref. 26, the transfer matrix $\tilde{t}_{1}$ in the basis of nonflux-normalized eigenfunctions (13) is of the blockdiagonal form,

$$
\tilde{t}_{1}=\left(\begin{array}{cccc}
g_{1} & \tilde{g}_{2} & 0 & 0 \\
\tilde{g}_{3} & g_{4} & 0 & 0 \\
0 & 0 & h_{1} & \tilde{h}_{2} \\
0 & 0 & \tilde{h}_{3} & h_{4}
\end{array}\right) .
$$

With $\omega_{\rho}=\cos \left(\zeta_{\rho}^{\uparrow}-\zeta_{\rho}^{\downarrow}\right)$, where $\zeta_{\rho}^{\sigma}=\gamma_{\rho}^{\sigma} / 2$, the entries for counterclockwise propagation read

$$
\begin{gathered}
g_{1}=\frac{1}{\omega_{+}}\left(e^{i n_{+}^{\uparrow} \pi} \cos \zeta_{+}^{\uparrow} \cos \zeta_{+}^{\downarrow}+e^{i n_{+}^{\downarrow} \pi} \sin \zeta_{+}^{\uparrow} \sin \zeta_{+}^{\downarrow}\right), \\
\tilde{g}_{2}=\frac{e^{-i \varphi_{t}}}{\omega_{+}}\left(e^{i n_{+}^{\uparrow} \pi}-e^{i n_{+}^{\downarrow} \pi}\right) \cos \zeta_{+}^{\uparrow} \sin \zeta_{+}^{\downarrow}, \\
\tilde{g}_{3}=\frac{-e^{i \varphi_{t}}}{\omega_{+}}\left(e^{i n_{+}^{\dagger} \pi}-e^{i n_{+}^{\downarrow} \pi}\right) \sin \zeta_{+}^{\uparrow} \cos \zeta_{+}^{\downarrow}, \\
g_{4}=\frac{-1}{\omega_{+}}\left(e^{i n_{+}^{\uparrow} \pi} \sin \zeta_{+}^{\uparrow} \sin \zeta_{+}^{\downarrow}+e^{i n_{+}^{\downarrow} \pi} \cos \zeta_{+}^{\uparrow} \cos \zeta_{+}^{\downarrow}\right) .
\end{gathered}
$$

Similar expressions apply to the clockwise propagating waves,

$$
\begin{gathered}
h_{1}=\frac{1}{\omega_{-}}\left(e^{i n_{-}^{\uparrow} \pi} \cos \zeta_{-}^{\uparrow} \cos \zeta_{-}^{\downarrow}+e^{i n_{-}^{\downarrow}} \sin \zeta_{-}^{\uparrow} \sin \zeta_{-}^{\downarrow}\right), \\
\tilde{h}_{2}=\frac{-e^{-i \varphi_{t}}}{\omega_{-}}\left(e^{i n_{-}^{\uparrow} \pi}-e^{i n_{-}^{\downarrow} \pi}\right) \cos \zeta_{-}^{\uparrow} \sin \zeta_{-}^{\downarrow}, \\
\widetilde{h}_{3}=\frac{e^{i \varphi_{t}}}{\omega_{-}}\left(e^{i n_{-}^{\uparrow} \pi}-e^{i n_{-}^{\downarrow} \pi}\right) \sin \zeta_{-}^{\uparrow} \cos \zeta_{-}^{\downarrow}, \\
h_{4}=\frac{-1}{\omega_{-}}\left(e^{i n_{-}^{\uparrow} \pi} \sin \zeta_{-}^{\uparrow} \sin \zeta_{-}^{\downarrow}+e^{i n_{-}^{\downarrow} \pi} \cos \zeta_{-}^{\uparrow} \cos \zeta_{-}^{\downarrow}\right) .
\end{gathered}
$$

We obtain $t_{1}$ from relation (34) after introducing the square root of the velocity ratios (obtained from the argument of particle conservation, or through direct computation) $\nu_{\rho}=\sqrt{v_{\rho}^{\pi} / v_{\rho}^{\Downarrow}}=\sqrt{\tan \zeta_{\rho}^{\top} / \tan \zeta_{\rho}^{\downarrow}}$ as

$$
t_{1}=\left(\begin{array}{cccc}
g_{1} & g_{2} & 0 & 0 \\
g_{3} & g_{4} & 0 & 0 \\
0 & 0 & h_{1} & h_{2} \\
0 & 0 & h_{3} & h_{4}
\end{array}\right)
$$

where $g_{2}=\nu_{+} \tilde{g}_{2}, g_{3}=\nu_{+}^{-1} \tilde{g}_{3}, h_{2}=\nu_{-} \widetilde{h}_{2}$, and $h_{3}=\nu_{-}^{-1} \widetilde{h}_{3}$.

The same algebra performed for the lower arm yields

$$
t_{2}=\left(\begin{array}{cccc}
g_{1} & -g_{2} & 0 & 0 \\
-g_{3} & g_{4} & 0 & 0 \\
0 & 0 & h_{1} & -h_{2} \\
0 & 0 & -h_{3} & h_{4}
\end{array}\right) .
$$



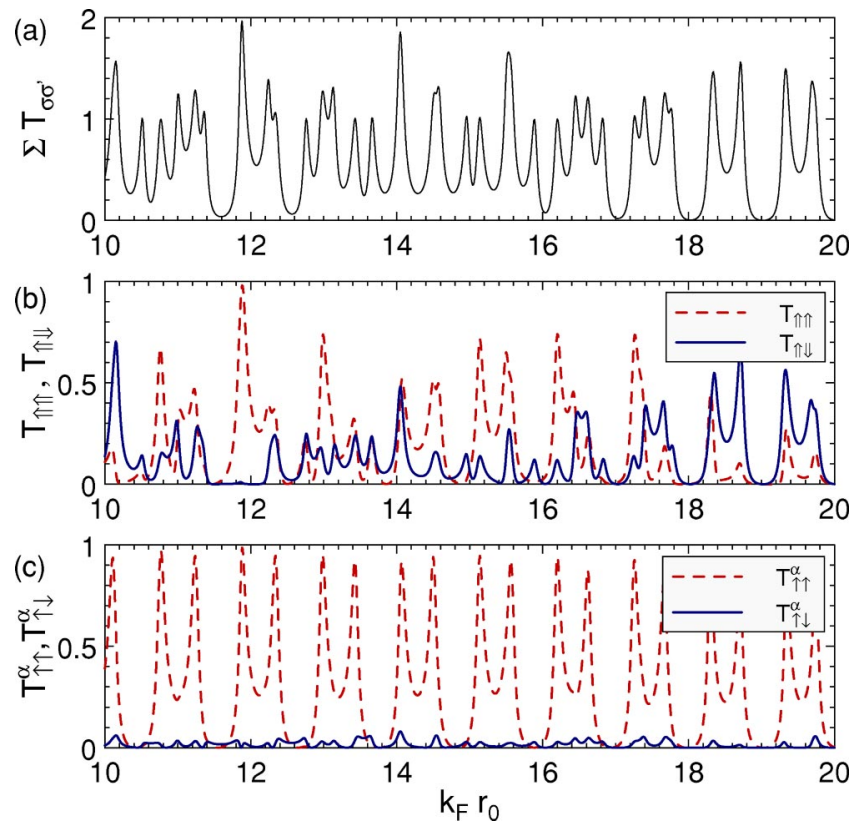

FIG. 4. (a) Dimensionless conductance $T=\Sigma_{\sigma, \sigma^{\prime}} T_{\sigma \sigma^{\prime}}$ and partial contributions in (b) the $S_{z}$ basis and (c) the $S_{\alpha}$ basis vs the scaled Fermi momentum $k_{F} r_{0}$ of the incident electrons $\left[g^{*}\right.$ $=1, m^{*}=m_{0}$, coupling strength $\varepsilon=0.25$, tilt angle $\alpha=\arctan 2$ $\approx 63.4^{\circ}$, adiabaticity parameter between $Q=3.35$ (for $k_{F} r_{0}=10$ ) and $Q=1.68$ (for $k_{F} r_{0}=20$ ), field texture parameter $\varphi_{t}=\pi / 2$ ]; corresponding to magnetic-field components $B_{r}=0, \tilde{\mu} B_{\varphi}$ $=30 \phi_{0} /\left(\pi r_{0}^{2}\right), \tilde{\mu} B_{z}=15 \phi_{0} /\left(\pi r_{0}^{2}\right)$. Shown are the diagonal and off-diagonal partial transmissions for electron initially in the state $|\uparrow\rangle$, parallel to the field. For all values of $k_{F} r_{0}$ we are in an (almost) adiabatic situation, as confirmed by the small spin-flip probabilities in $S_{\alpha}$ basis in panel (c).

Now we have provided all ingredients to calculate transmission amplitudes and probabilities according to Eqs. (25), (31), and (32) for any desired field configuration that respects rotational symmetry.

\section{Transformations in spin space}

So far, we always used the $S_{z}$ basis to express the spin states needed to calculate the transfer matrices. Here, the transmission probabilities $t_{\Uparrow \Downarrow}, t_{\Downarrow \Uparrow}$ for spin-flip processes are nonzero for a tilted magnetic field even in the adiabatic limit. We recall that there are no transitions between the propagating states $|\uparrow(\varphi)\rangle,|\downarrow(\varphi)\rangle$ in this case. This is, of course, no contradiction since $|\uparrow(\varphi)\rangle,|\downarrow(\varphi)\rangle$ represent spins aligned with the magnetic field, given in $S_{z}$ basis by Eq. (3).

Alternatively, one can consider transmission amplitudes in the local $S_{\alpha}$ basis (3). The new transmission amplitudes $t_{\uparrow \uparrow}^{\alpha}, t_{\uparrow \downarrow}^{\alpha}, t_{\downarrow \uparrow}^{\alpha}, t_{\downarrow \downarrow}^{\alpha}$ that replace the ones with respect to the $S_{z}$ basis in the matrix $t$, Eq. (32), are obtained by performing the appropriate projections, e.g.,

$$
t_{\uparrow \uparrow}^{\alpha}=\langle\uparrow(\varphi=\pi)|t| \uparrow(\varphi=0)\rangle .
$$

In Fig. 4 we show an example for the full as well as the partial transmission probabilities in the $S_{z}$ and $S_{\alpha}$ bases. Figure 4(a) illustrates the oscillations in the dimensionless con- ductance as a function of the scaled Fermi momentum $k_{F} r_{0}$ $=\widetilde{E}_{F}^{1 / 2}$ for parameters chosen in the adiabatic regime $(Q$ $>1$ ). The diagonal and off-diagonal transmission probabilities in $S_{z}$ and $S_{\alpha}$ bases are shown in Figs. 4(b) and 4(c) for electrons entering the ring with initial spin parallel to the magnetic field (state $|\uparrow\rangle$ ). Note the differences between the $S_{z}$ and $S_{\alpha}$ representations-the spin-switching components $T_{\uparrow \downarrow}^{\alpha}, T_{\downarrow \uparrow}^{\alpha}$ only vanish in $S_{\alpha}$ basis as expected under adiabatic conditions. We point out that the off-diagonal partial transmissions coincide due to the reflection symmetry of the system about an axis perpendicular to the leads through the center of the ring.

\section{GEOMETRIC PHASES IN THE MAGNETOCONDUCTANCE}

Here and in the following section we discuss the influence of geometric and Aharonov-Bohm phases on the magnetoconductance through the $1 \mathrm{D}$ rings. In this section we consider an inhomogeneous magnetic field generated by a central micromagnet and study the magnetoconductance as the Fermi energy of the incident electrons or the magnetic field in $z$ direction is varied by applying an external magnetic field. In Sec. V we will concentrate on in-plane magnetic fields $(\alpha \approx \pi / 2)$ and use $B_{z}$ as a small control field.

Our main motivation to study rotational invariant configurations of the magnetic field is the experimental realization of a crownlike magnetic field by means of a central micromagnet as reported in Ref. 22. The magnetic field of the cylinder-shaped Dysprosium micromagnet can be approximated by a dipole field at the position of the ring. ${ }^{28}$ It contains both a radial and a $z$ component, $\vec{B}_{\mathrm{M}}=B_{r} \vec{e}_{r}+B_{z, \mathrm{M}} \vec{e}_{z}$. The total magnetic field is obtained after adding the homogeneous magnetic field $\vec{B}_{\text {ext }}=B_{\text {ext }} \vec{e}_{z}$ in $z$ direction, $\vec{B}=\vec{B}_{\mathrm{M}}$ $+\vec{B}_{\text {ext }}$. Both $\vec{B}_{\mathrm{M}}$ and $\vec{B}_{\text {ext }}$ contribute to the Aharonov-Bohm flux through the ring, and we denote the magnetic flux due to the homogeneous field by $\phi_{\mathrm{ext}}^{\mathrm{AB}}=\pi r_{0}^{2} B_{\text {ext }}$. The strength of the magnetic field depends on the premagnetization procedure, but is constant during the magnetoconductance measurement where the homogeneous magnetic field perpendicular to the ring is varied. The magnetization of the micromagnet is characterized by the parameters

$$
Q_{M}=\frac{\tilde{\mu}\left|\vec{B}_{M}\right|}{k_{F} r_{0}}, \quad M^{\mathrm{AB}}=-\frac{\pi r_{0}^{2} B_{z, \mathrm{M}}}{\phi_{0}},
$$

where $Q_{M}$ is introduced in analogy to Eq. (4) as adiabaticity parameter for the micromagnet for vanishing external flux, $\phi_{\mathrm{ext}}^{\mathrm{AB}}=0$.

The results for the calculated magnetoconductance are shown in Fig. 5 for three different degrees of adiabaticity, $Q_{M}=0.4$ [Fig. 5(b)], 1 [Fig. 5(c)], and 10 [Fig. 5(d)]. This parameter is adjusted by the proper choice of the effective gyromagnetic ratio $g^{*}$ and the effective mass $m^{*}$, leaving the strength of the micromagnet constant at $M^{\mathrm{AB}}=5$. The connection to a specific material system is then provided by choosing the value for $Q_{M}$ that corresponds to the product $g^{*} m^{*}$. We follow the geometry described in Ref. 22 and 

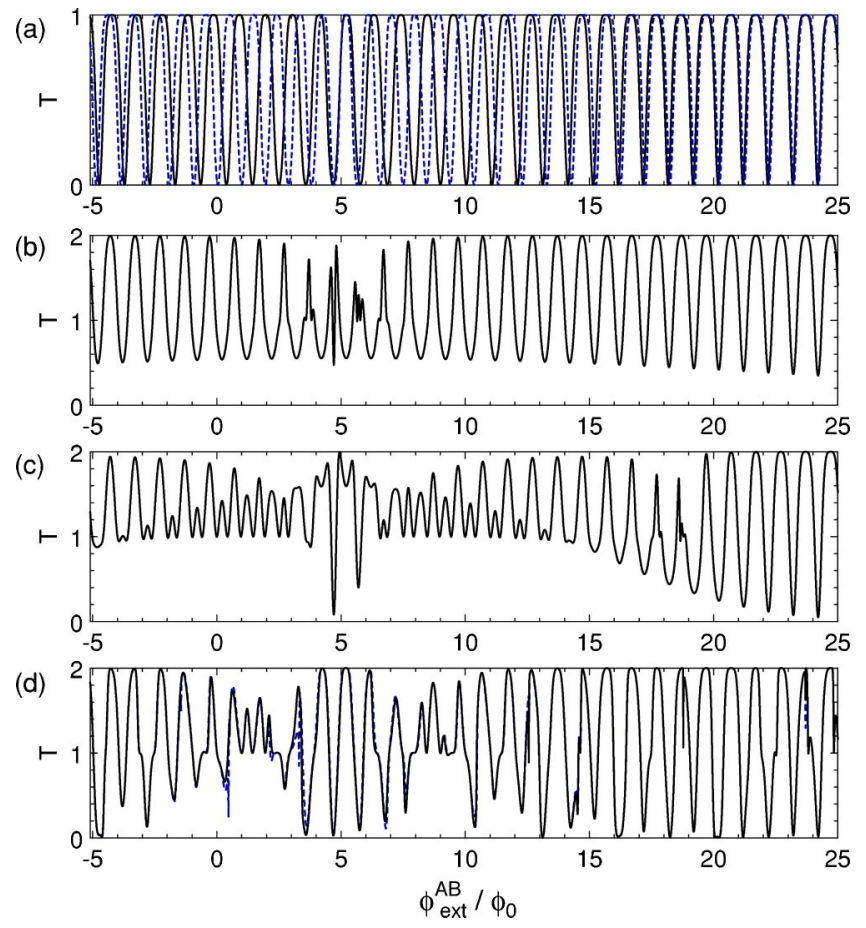

FIG. 5. Magnetoconductance for an Aharonov-Bohm device with a central micromagnet $\left(\varphi_{t}=0, M^{\mathrm{AB}}=5\right)$ under variation of the external flux $\phi_{\mathrm{ext}}^{\mathrm{AB}}$. In (a) we show the dimensionless conductance for independent electron gases ||$\uparrow\rangle\rangle$ (solid curve) and $\| \downarrow\rangle\rangle$ (dashed curve) as in Ref. 27, but taking the Zeeman energy and Berry phase into account. In (d), the sum of the two contributions is shown as dashed line. The dimensionless conductance for spinful electrons is given in panels (b) $-(\mathrm{d})$; (b) diabatic regime $\left(Q_{M}\right.$ $=0.4)$, (c) intermediate case $\left(Q_{M}=1\right)$ where effects due to geometrical phases become visible, and (d) adiabatic limit $\left(Q_{M}=10\right)$, which is dominated by interference effects due to different Berry phases for the electron gases ||$\uparrow\rangle\rangle$ and ||$\downarrow\rangle\rangle$. We point out the similarity to the magnetoconductance measured in the experiment of Ref. 21. The effect of geometric phases is always lost for dominating external field, $\phi_{\text {ext }}^{\mathrm{AB}} \gg M^{\mathrm{AB}} \phi_{0}$, because then $\alpha \rightarrow 0$.

choose the radius of the ring as $r_{0}=500 \mathrm{~nm}$. The 2DEG is placed in a plane lying $150 \mathrm{~nm}$ above the central plane of the Dysprosium. We assume maximal coupling between ring and leads, $\varepsilon=0.5$.

For comparison, we show in Fig. 5(a) the result of Büttiker et al. ${ }^{27}$ for spin-independent transport, with the wellknown Aharonov-Bohm oscillations as the homogeneous magnetic field is varied. However, here we have taken into account the Zeeman splitting of the energy and the influence of the Berry phase (assuming an adiabatic situation), as is discussed at the end of Sec. III A. In Figs. 5(b)-5(d) the strongest deviations from Aharonov-Bohm-like oscillations are seen around $\phi_{\mathrm{ext}}^{\mathrm{AB}} / \phi_{0}=M^{\mathrm{AB}}=5$, indicating the importance of geometric phases there. In fact, this corresponds to the situation where the external flux $\phi_{\mathrm{ext}}^{\mathrm{AB}}$ cancels the magnetic flux due to the micromagnet such that the nonuniform field of the micromagnet becomes maximally important. The strong interference effects around $\phi_{\mathrm{ext}}^{\mathrm{AB}} / \phi_{0}=M^{\mathrm{AB}}$ stem from the slightly different oscillation frequencies of the $|\uparrow\rangle$ and $|\downarrow\rangle$ electrons due to the Berry phase. Regular Aharonov-
Bohm-like oscillations are recovered as the external magnetic field $B_{\text {ext }}$ becomes dominant, which requires a smaller value of this field in the diabatic situation of Fig. 5(b), when compared to the intermediate case of Fig. 5(c) or to the adiabatic case of Fig. 5(d). Note that the curves in Figs. 5(b)$5(\mathrm{~d})$ are not quite symmetric about the line $\phi_{\mathrm{ext}}^{\mathrm{AB}} / \phi_{0}=M^{\mathrm{AB}}$ (corresponding to a vanishing overall Aharonov-Bohm flux $\left.\phi^{\mathrm{AB}}=0\right)$, since there the angles $\gamma_{\rho}^{\sigma}$ still change monotonically.

We point out that there exist two adiabatic limits: one for dominating field of the micromagnet (parametrized by $Q_{M}$ ), and the other one for dominating external field $B_{\text {ext }}$, which is always reached for sufficiently large $\left|\phi_{\text {ext }}^{\mathrm{AB}}\right| \gg\left|M^{\mathrm{AB}}\right| \phi_{0}$. In the sequence of Figs. 5(b) $-5(\mathrm{~d})$, the adiabaticity is increased with respect to the first limit, i.e., the magnetic energy due to the micromagnet (at zero external flux $\phi_{\text {ext }}^{\mathrm{AB}}$ ) becomes large in comparison to the Fermi energy of the electrons. As a result, the magnetoconductance in Fig. 5(d) is well described by the sum of the two curves for the electron gases $\| \uparrow\rangle\rangle$ and $\| \downarrow\rangle\rangle$ in Fig. 5(a), which is indicated by the dashed curve in Fig. 5(d).

The above-mentioned experiment ${ }^{22}$ was performed under rather diabatic conditions [similar to those of Fig. 5(b)], which are not favorable for the detection of geometric phase effects. Accordingly, it was found that the experimental observations could not be accounted for by geometric phases. However, evidence for geometric phase effects in electronic ring structures should be possible in more adiabatic regimes, that could, e.g., be achieved using stronger micromagnets, appropriately arranged ferromagnetic particles ${ }^{29}$ or by exploiting the spin-orbit interaction. ${ }^{21,30}$ The magnetoconductance measured in the experiment of Ref. 21 for a singly connected InAs ring with a radius $250 \mathrm{~nm}$ and a spin-orbit induced magnetic field strongly resembles our results presented in Figs. 5(c) and 5(d).

\section{IN-PLANE MAGNETIC FIELD}

\section{A. Aharonov-Bohm ring as a spin switch}

We will now consider a ring that is subject to an in-plane magnetic field, which can be either circular, radial, or a rotationally invariant combination of the two. In experiments, such a field could be generated, e.g., by a current through the ring. ${ }^{23}$ We recently reported a spin-flip effect for this magnetic-field texture, ${ }^{1,24}$ by which one can change the spin polarization of the transmitted electrons with a small (external) Aharonov-Bohm flux through the ring. The spindependent transmittance is periodic in the applied magnetic flux, with a period of one flux quantum $\phi_{0}$. In particular, the polarization state of polarized electrons can be inverted by altering this magnetic control flux by $\phi_{0} / 2$, which might become interesting for future spintronic devices. In paper II of this series (Ref. 1) we investigated in detail necessary conditions for the spin-flip effect to hold.

For an in-plane magnetic field, $\alpha=\pi / 2$, we find a high symmetry between the clockwise and counterclockwise propagating waves. This becomes manifest in simple relations between the angles $\gamma_{\rho}^{\sigma}$ entering the spin states (13) and 
the orbital quantum numbers $n_{\rho}^{\sigma}$ which solve Eq. (12). For the case with no magnetic flux through the ring, $\phi^{\mathrm{AB}}=0$, we find

$$
\begin{array}{cc}
\gamma_{+}^{\uparrow}=\gamma_{-}^{\downarrow}, & n_{+}^{\uparrow}=-n_{-}^{\downarrow}-1, \\
\gamma_{+}^{\downarrow}=\gamma_{-}^{\uparrow}, \quad n_{+}^{\downarrow}=-n_{-}^{\uparrow}-1 .
\end{array}
$$

This simplifies the structure of the transfer matrices $t_{1}, t_{2}$, cf. Eqs. (38) and (39), since we can now use the relations

$$
\begin{array}{ll}
g_{1}=h_{4}^{*}, & g_{2}=h_{3}^{*}, \\
g_{4}=h_{1}^{*}, & g_{3}=h_{2}^{*} .
\end{array}
$$

The number of variables can be reduced further if we specify the field texture parameter $\varphi_{t}$ in order to relate $g_{2}$ and $g_{3}$. We find $g_{2}=-(+) g_{3}$ in the case of a radial (circular) magnetic field, $\varphi_{t}=0 \quad\left(\varphi_{t}=\pi / 2\right)$. These relations still hold when an integer number of flux quanta penetrate the ring, as can be seen from Eqs. (36) and (37), because this corresponds to changes of $n$ by an integer-and an overall sign change, where applicable, does not change the transmission amplitude. Together with the definitions (28)-(30), (38), and (39), the relations in Eq. (43), and the unitarity and symmetry of the matrices $t_{1}, t_{2}$, we now can give a rigorous analytical proof of the above-mentioned spin-switch effect.

Evaluating the matrix $P^{\prime-1}$ given by Eq. (31) in a straightforward computation for $\phi^{\mathrm{AB}} / \phi_{0} \in \mathbb{N}$, we obtain for a circular magnetic field, $g_{2}=g_{3}$,

$$
P^{\prime-1}=\frac{1}{2 b}\left(\begin{array}{cccc}
\delta_{1} & \delta_{2} & \delta_{3} & 0 \\
\delta_{2} & -\delta_{1} & 0 & \delta_{3} \\
\delta_{4} & 0 & \delta_{1} & \delta_{2} \\
0 & \delta_{4} & \delta_{2} & -\delta_{1}
\end{array}\right)
$$

with

$$
\begin{gathered}
\delta_{1}=\left(g_{1}-g_{4}\right)(a+b)-\left(g_{1}^{*}-g_{4}^{*}\right), \\
\delta_{2}=-2\left(g_{2}(a+b)+g_{3}^{*}\right), \\
\delta_{3}=-\left(g_{1}+g_{4}\right)+\left(g_{1}^{*}+g_{4}^{*}\right), \\
\delta_{4}=-\left(g_{1}+g_{4}\right)(a+b)^{2}+\left(g_{1}^{*}+g_{4}^{*}\right) .
\end{gathered}
$$

The inversion of this matrix yields

$$
\begin{gathered}
P_{13}^{\prime}=P_{24}^{\prime}=2 b \frac{\delta_{3}}{\delta_{3} \delta_{4}-\left(\delta_{1}^{2}+\delta_{2}^{2}\right)}, \\
P_{14}^{\prime}=P_{23}^{\prime}=0,
\end{gathered}
$$

and these results carry over to the transmission amplitudes (32) in the $S_{z}$ basis,

$$
t_{\Uparrow \Uparrow}=t_{\Downarrow \Downarrow} \quad \text { and } \quad t_{\Uparrow \Downarrow}=t_{\Downarrow \Uparrow}=0 .
$$

We also can express the transmission probabilities in the $S_{\alpha}$ basis according to Eq. (40), yielding

$$
t_{\uparrow \uparrow}^{\alpha}=t_{\downarrow \downarrow}^{\alpha}=t_{\Uparrow \downarrow}=t_{\Downarrow \Uparrow}=0, \quad t_{\uparrow \downarrow}^{\alpha}=t_{\downarrow \uparrow}^{\alpha}=t_{\Uparrow \Uparrow}=t_{\Downarrow \Downarrow},
$$

that is, the role of the diagonal ( $\Uparrow$ and $\Downarrow \Downarrow)$ and nondiagonal elements ( $\Downarrow$ and $\Downarrow \Uparrow)$ is just interchanged when switching between $S_{z}$ and $S_{\alpha}$ bases.

The result (46) states that only electrons that $f l i p^{31}$ their spin with respect to the magnetic field are transmitted through the ring. Moreover, the properties (46) of the transmission amplitudes remain valid for any in-plane magneticfield configuration. This explains the numerical findings for $2 \mathrm{D}$ rings in Refs. 1 and 24 . Note that the spin-flip effect holds also in the adiabatic limit. Since this counteracts the adiabatic spin alignment, the dimensionless conductance tends to zero in this limit.

We now turn to the case where half a flux quantum penetrates the ring, such that there is an Aharonov-Bohm flux $\phi^{\mathrm{AB}}=\phi_{0} / 2$. We assume the magnetic field at the position of the ring to remain unchanged, as in the case of an $\mathrm{AB}$ flux generated by a solenoid (rather than by a homogeneous $B_{z}$ component). The quantum number $n$ is then reduced by half an integer, cf. Eqs. (11), and Eq. (43) has to be replaced by

$$
\begin{array}{ll}
g_{1}=-h_{4}^{*}, & g_{2}=-h_{3}^{*}, \\
g_{4}=-h_{1}^{*}, & g_{3}=-h_{2}^{*} .
\end{array}
$$

These relations also directly follow from Eqs. (36) and (37), since the coefficients are multiplied by a factor $e^{-i \pi / 2}$ compared to the values obtained for $\phi^{\mathrm{AB}}=0$. Hence we can write

$$
t_{j}^{\phi_{0} / 2}=-i t_{j}^{0},
$$

where $t_{j}^{0}$ are the transfer matrices for $\phi^{\mathrm{AB}}=0$. Accordingly, we now find

$$
\begin{aligned}
P^{\prime}\left(\phi_{0} / 2\right) & =\left[-i N_{2} t_{2}^{0} N_{1}-\frac{1}{-i} N_{1}^{-1} t_{1}^{0-1} N_{2}^{-1}\right]^{-1} \\
& =-\frac{1}{i}\left[N_{2} t_{2}^{0} N_{1}+N_{1}^{-1} t_{1}^{0-1} N_{2}^{-1}\right]^{-1} .
\end{aligned}
$$

For a circular magnetic field $\left(g_{2}=g_{3}\right)$ this results in

$$
\left[P^{\prime}\left(\phi_{0} / 2\right)\right]^{-1}=\frac{1}{2 b}\left(\begin{array}{cccc}
\delta_{1}^{\prime} & 0 & \delta_{2}^{\prime} & \delta_{3}^{\prime} \\
0 & -\delta_{1}^{\prime} & \delta_{3}^{\prime} & -\delta_{2}^{\prime} \\
\delta_{4}^{\prime} & \delta_{5}^{\prime} & \delta_{1}^{\prime} & 0 \\
\delta_{5}^{\prime} & -\delta_{4}^{\prime} & 0 & -\delta_{1}^{\prime}
\end{array}\right),
$$

with

$$
\begin{gathered}
\delta_{1}^{\prime}=\left(g_{1}+g_{4}\right)(a+b)+\left(g_{1}^{*}+g_{4}^{*}\right), \\
\delta_{2}^{\prime}=-\left(g_{1}-g_{4}\right)-\left(g_{1}^{*}-g_{4}^{*}\right), \\
\delta_{3}^{\prime}=2\left(g_{2}-g_{3}^{*}\right), \\
\delta_{4}^{\prime}=-\left(g_{1}-g_{4}\right)(a+b)^{2}-\left(g_{1}^{*}-g_{4}^{*}\right), \\
\delta_{5}^{\prime}=2\left(g_{2}(a+b)^{2}-g_{3}^{*}\right),
\end{gathered}
$$


where the $g_{i}$ are the same as for $\phi^{\mathrm{AB}}=0$. Inverting this matrix one obtains

$$
\begin{gathered}
P_{13}^{\prime}\left(\phi_{0} / 2\right)=-P_{24}^{\prime}\left(\phi_{0} / 2\right)=2 b \frac{-\delta_{1}^{\prime 2} \delta_{2}^{\prime}+\left(\delta_{2}^{\prime 2}+\delta_{3}^{\prime 2}\right) \delta_{4}^{\prime}}{\mathcal{N}}, \\
P_{14}^{\prime}\left(\phi_{0} / 2\right)=P_{23}^{\prime}\left(\phi_{0} / 2\right)=2 b \frac{-\delta_{1}^{\prime 2} \delta_{3}^{\prime}+\left(\delta_{2}^{\prime 2}+\delta_{3}^{\prime 2}\right) \delta_{5}^{\prime}}{\mathcal{N}}
\end{gathered}
$$

with the common denominator

$$
\mathcal{N}=\delta_{1}^{\prime 4}+\left(\delta_{2}^{\prime 2}+\delta_{3}^{\prime 2}\right)\left({\delta_{4}^{\prime}}^{2}+\delta_{5}^{\prime 2}\right)-2 \delta_{1}^{\prime 2}\left(\delta_{2}^{\prime} \delta_{4}^{\prime}+\delta_{3}^{\prime} \delta_{5}^{\prime}\right) .
$$

Again, this carries over to the transmission amplitudes (32), and the result in $S_{z}$ basis reads

$$
t_{\Uparrow \Uparrow}^{\phi_{0} / 2}=-t_{\Downarrow \Downarrow}^{\phi_{0} / 2} \quad \text { and } \quad t_{\Uparrow \Downarrow}^{\phi_{0} / 2}=t_{\Downarrow \Uparrow}^{\phi_{0} / 2},
$$

where all quantities are nonzero, in contrast to the result for the nondiagonal transmission amplitudes in the case $\phi^{\mathrm{AB}}$ $=0$. Transformation to the $S_{\alpha}$ basis, however, yields

$$
{ }^{\alpha} t_{\uparrow \uparrow, \downarrow \downarrow}^{\phi_{0 / 2}} \neq 0, \quad{ }^{\alpha} t_{\uparrow \downarrow, \downarrow \uparrow}^{\phi_{0 / 2}}=0 .
$$

This result applies whenever the Aharonov-Bohm flux equals half an integer number of flux quanta $\phi_{0}$, i.e., $\phi^{\mathrm{AB}}$ $= \pm \phi_{0} / 2, \pm 3 \phi_{0} / 2, \ldots$. The physical meaning of this result is that in the presence of half a flux quantum only electrons that keep their spin direction with respect to the magnetic field during transport are transmitted-precisely the opposite of what we found for $\phi^{\mathrm{AB}}=0$. This opens the possibility of controlling the transmission of (polarized) electrons by varying the number of flux quanta penetrating the ring: For $\phi^{\mathrm{AB}} / \phi_{0}$ integer, only spin-flipping electrons are transmitted, whereas for half-integer number of flux quanta only electrons keeping their spin polarization can be found in the exiting lead. For an illustration of these two situations see Figs. 6(a) and 6(c). For magnetic fluxes in between, the situation is intermediate with transport in all channels, see Fig. 6(b) with $\phi^{\mathrm{AB}}=\phi_{0} / 4$.

Remember that in the derivation of the spin-flip effect we have assumed the Aharonov-Bohm flux to cause no change in the angle $\alpha$ of the magnetic field $\vec{B}$ with the $z$ axis. Therefore, in realistic situations where the magnetic control flux might originate from a homogeneous magnetic-field component $B_{z}$ in $z$ direction, which also acts on the electronic spin in the ring, we have to expect deviations from the result (51). In order to test the sensitivity of the spin-flip effect, we indeed followed this procedure in Fig. 6, and find that for large in-plane magnetic fields used there the influence of $B_{z}$ is negligible.

\section{B. Energy-averaged conductance}

So far we discussed the spin-flip effect in terms of rapidly oscillating (total and partial) dimensionless conductances. Energy averaging these quantities, while keeping the degree of adiabaticity $Q$ constant, cancels out the oscillations and reveals inherent features that we will study in the following.

For an example of the averaged dimensionless conductance at fixed $Q$ and variable Aharonov-Bohm flux $\phi^{\mathrm{AB}}$ see
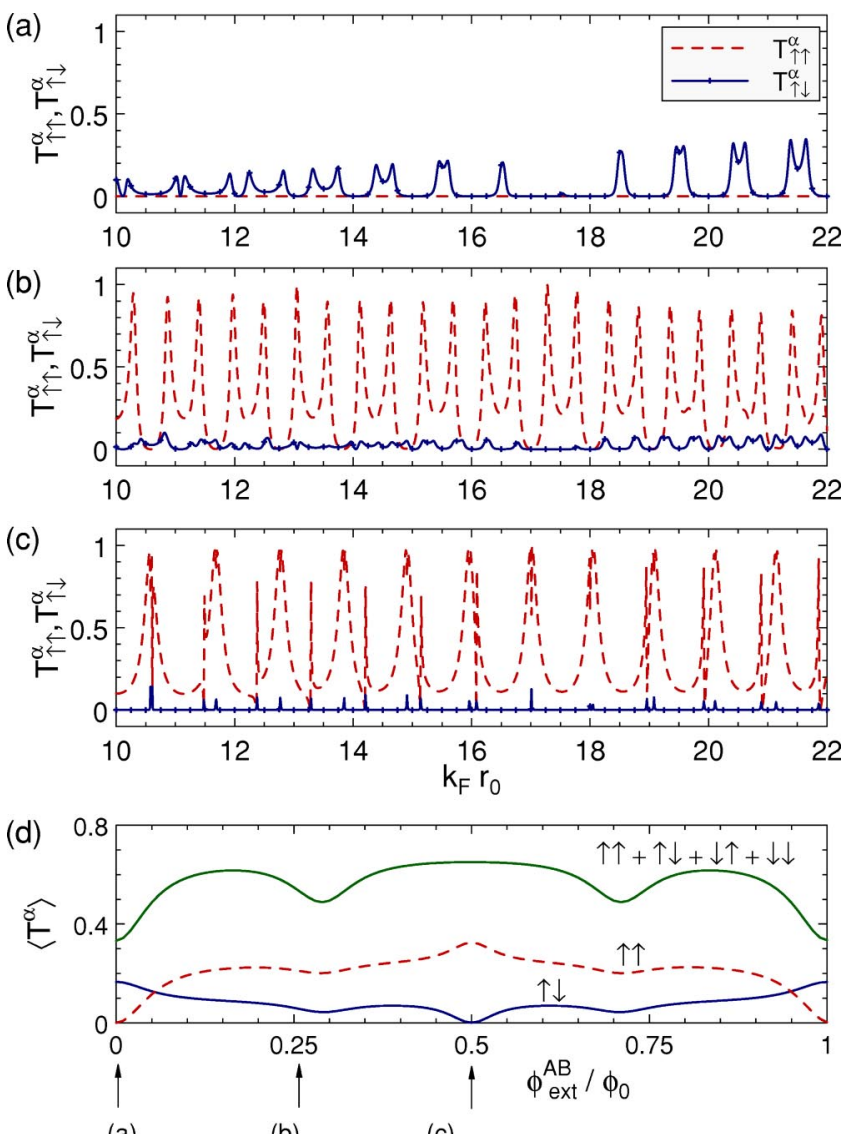

(a)

(b)

(c)

FIG. 6. Partial transmission probabilities vs scaled momentum $k_{F} r_{0}$ of the incident electrons $\left(g^{*}=1, m^{*}=m_{0}, \varepsilon=0.25\right)$ at fixed strength of the circular magnetic field $\tilde{\mu} B_{\varphi}=30 \phi_{0} /\left(\pi r_{0}^{2}\right) \quad(Q$ $\approx 2$ ). (a) $\phi^{\mathrm{AB}}=0$ : (nearly) zero transmission for the smallest $k_{F} r_{0}$ shown (due to the Berry phase in the adiabatic situation) and around $k_{F} r_{0}=\tilde{\mu} B / \sqrt{3} \approx 17.32$. Note that the transmission is provided only by the spin-flipping channels, i.e., only $T_{\uparrow \downarrow}^{\alpha}$ and $T_{\downarrow \uparrow}^{\alpha}$ are nonzero. (c) $\phi^{\mathrm{AB}}=\phi_{0} / 2$ : opposite situation such that now $T_{\uparrow \uparrow}^{\alpha}$ and $T_{\downarrow \downarrow}^{\alpha}$ are nonzero. Finite values for the spin-flipping channels arise because the magnetic flux was created by a homogeneous field $B_{z}$ affecting the angle $\alpha$. (b) For intermediate values of the Aharonov-Bohm flux (here, $\left.\phi^{\mathrm{AB}}=\phi_{0} / 4\right)$, transport occurs in all channels. Hence, for polarized incident electrons, the spin can be switched by applying an Aharonov-Bohm flux of half a flux quantum. This is shown in (d) for the energy-averaged transmission vs control flux. The situations shown in (a)-(c) are marked by arrows.

Fig. 6(d). The systematic dependence on this parameter and the coupling strength $\varepsilon$ for $\phi^{\mathrm{AB}}=0$ is shown in Fig. 7. In the adiabatic limit $(Q \rightarrow \infty)$, the dimensionless conductance approaches zero due to the effect of the Berry phase. In the other, diabatic, limit $(Q \rightarrow 0)$, the numerical data agree with the analytical result (20) that was multiplied by 2 to account for two open channels.

Most interestingly, there are regions where $\langle T\rangle$ drops to zero that are not caused by geometric-phase effects. In order to investigate what happens there, we look at the nonvanishing transmission amplitudes (45), in particular at the numerator $\delta_{3}=g_{1}^{*}-g_{1}+g_{4}^{*}-g_{4}=-2 i \operatorname{Im}\left(g_{1}+g_{4}\right)$. With the expressions for $g_{1}, g_{4}$ from Eq. (36), we find 


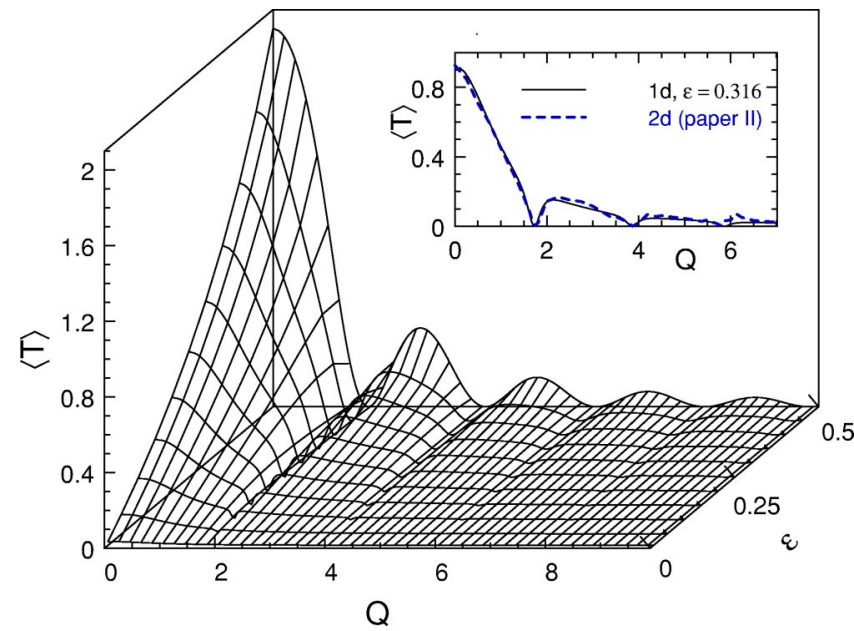

FIG. 7. Energy-averaged dimensionless conductance as a function of the adiabaticity parameter $Q$ for an in-plane circular magnetic field $\left(\phi_{t}=\alpha=\pi / 2\right)$ and vanishing magnetic flux $\phi^{\mathrm{AB}}=\phi_{\mathrm{ext}}^{\mathrm{AB}}$ $=0$. The tilted axis is the coupling parameter $\varepsilon$. The materialspecific parameters $g *$ for the gyromagnetic ratio and the effective mass $m^{*}$ are chosen here and in the following figures as $g^{*}=15$ and $m^{*}=0.023 m_{0}$ corresponding to InAs. The maximum $\langle T\rangle_{\max }$ of $\langle T\rangle$ is observed at $Q=0$, i.e., in the diabatic limit. It only depends on $\varepsilon$ and obeys the law $\langle T\rangle_{\max }=2 \varepsilon /(1-\varepsilon)$ where $0 \leqslant \varepsilon \leqslant 0.5$. Minima in $\langle T\rangle$ occur at $Q=\sqrt{4 m^{2}-1}(m \in \mathbb{N})$. The overall decay of $\langle T\rangle$ to zero in the adiabatic limit $Q \rightarrow \infty$ is an effect of the Berry phase. The inset shows a comparison between the results of the $2 \mathrm{D}$ model used in paper II and the $1 \mathrm{D}$ model with $\epsilon=0.316$.

$$
\delta_{3}=-2 i\left(\sin n_{+}^{\uparrow} \pi-\sin n_{+}^{\downarrow} \pi\right) \frac{\cos \left(\zeta_{+}^{\uparrow}+\zeta_{+}^{\downarrow}\right)}{\cos \left(\zeta_{+}^{\uparrow}-\zeta_{+}^{\downarrow}\right)},
$$

implying that $\delta_{3}$ goes to zero whenever the difference in the sine factor vanishes. Obviously, this occurs when $n_{+}^{\uparrow}$ and $n_{+}^{\downarrow}$ differ by an even integer number. Expanding the difference $\Delta n=n_{+}^{\uparrow}-n_{+}^{\downarrow}$ for small $\tilde{\mu} B / \widetilde{E}_{F}$, and using $\gamma_{+}^{\uparrow} \approx \gamma_{+}^{\downarrow}$, we have to solve the (Diophantic-like) equation

$$
\Delta n=\cos \frac{\gamma_{+}^{\uparrow}+\gamma_{+}^{\downarrow}}{2} Q \sin \frac{\gamma_{+}^{\uparrow}+\gamma_{+}^{\downarrow}}{2}
$$

to yield $\Delta n$ as an even integer-keeping in mind that the $\gamma_{\rho}^{\sigma}$ themselves depend on the $n$. The first zero is related to $\Delta n$ $=2$ with $\left(\gamma_{+}^{\uparrow}+\gamma_{+}^{\downarrow}\right) / 2=\pi / 3$, giving $Q=\sqrt{3}$ in accordance with the observation in Fig. 7. This value is consistent with the condition $\tilde{\mu} B / \widetilde{E}_{F} \ll 1$ as long as $\widetilde{E}_{F}^{1 / 2} \gg \sqrt{3}$. The further zeros in the averaged dimensionless conductance belong to $\Delta n=2 m$ (with the integer $m \geqslant 2$ ) and occur at ratios $Q$ $=\sqrt{4 m^{2}-1}$, as is clearly visible in Fig. 7 . Also note that the last factor in Eq. (52) accounts for the overall decay of $\langle T\rangle(Q)$, whereas the factor discussed above is responsible for the oscillations with respect to $Q$. The $Q$ dependence of $\langle T\rangle$ around the zeros also strongly depends on the coupling parameter $\varepsilon$; see Figs. 7 and 8 .
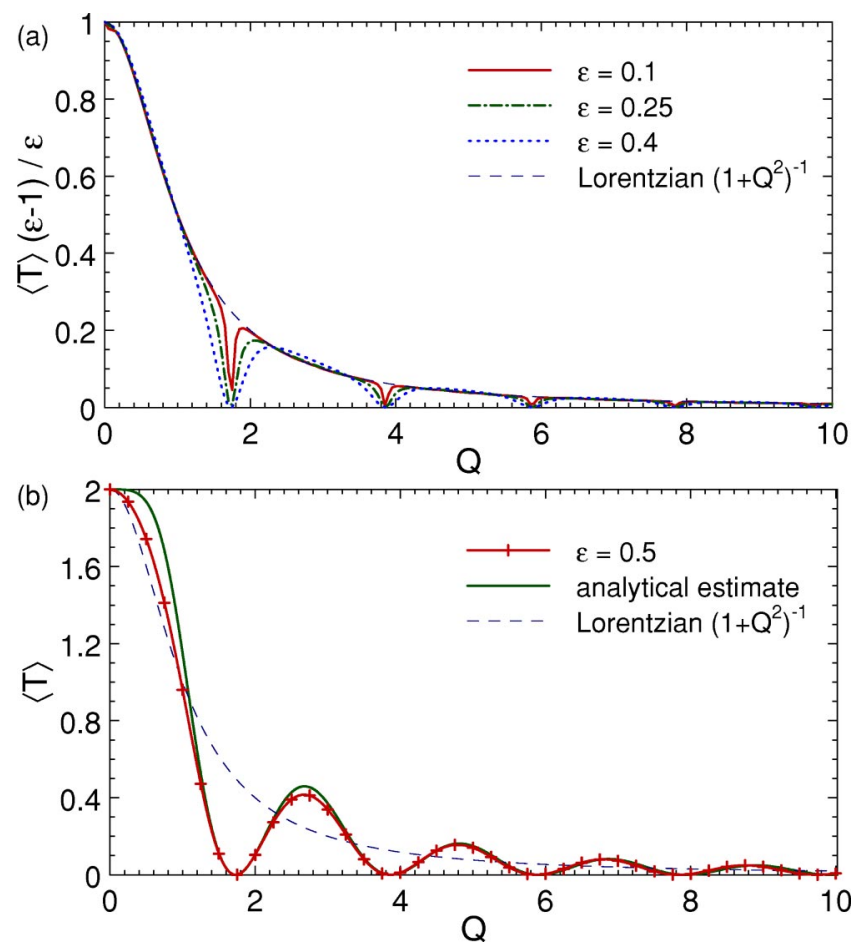

FIG. 8. (a) Energy-averaged dimensionless conductance $\langle T\rangle$ of Fig. 7, scaled by a factor $(1-\varepsilon) / \varepsilon$ for various coupling strengths $\varepsilon<0.4$. Even though the precise shape around the minima depends strongly on the value of $\varepsilon$ (in particular, it becomes singular for $\varepsilon$ $\rightarrow 0$ ), the overall decay away from the minima is very well described by the Lorentzian envelope function (60). (b) Energyaveraged dimensionless conductance for maximal coupling strength $\varepsilon=0.5$, in an in-plane (circular) magnetic field $\left(\varphi_{t}=\alpha=\pi / 2, \phi^{\mathrm{AB}}\right.$ $=0$ ). The exact result (crosses) is well described by the analytical expression, Eq. (59). For comparison, the Lorentzian decay is also shown.

We will now derive an approximate analytical expression for the averaged dimensionless conductance $\langle T\rangle$ for the case of maximal coupling strength $\varepsilon=0.5$. Equation (44) yields

$$
\begin{gathered}
\delta_{1}=-\left(g_{1}^{*}-g_{4}^{*}\right), \\
\delta_{2}=-2 g_{3}^{*}, \\
\delta_{3}=g_{1}^{*}-g_{1}+g_{4}^{*}-g_{4}, \\
\delta_{4}=g_{1}^{*}+g_{4}^{*} .
\end{gathered}
$$

For the denominator $\mathcal{N} \equiv \delta_{3} \delta_{4}-\left(\delta_{1}^{2}+\delta_{2}^{2}\right)$ in Eq. (45), we obtain

$$
\mathcal{N}=-\left(g_{1}+g_{4}\right)\left(g_{1}^{*}+g_{4}^{*}\right)+4 \operatorname{det} g^{*},
$$

with det $g=g_{1} g_{4}-g_{3}^{2}$. Expressing the $g_{i}$ through Eqs. (36), and substituting $\Delta n=n_{+}^{\uparrow}-n_{+}^{\downarrow}$, we arrive at

$$
\left(g_{1}+g_{4}\right)\left(g_{1}^{*}+g_{4}^{*}\right)=2 \frac{\cos ^{2}\left(\zeta_{+}^{\uparrow}+\zeta_{+}^{\downarrow}\right)}{\cos ^{2}\left(\zeta_{+}^{\uparrow}-\zeta_{+}^{\downarrow}\right)}(1-\cos \Delta n \pi),
$$




$$
\operatorname{det} g=-e^{i\left(n_{+}^{\uparrow}+n_{+}^{\downarrow}\right) \pi} .
$$

This finally leads to the analytical expression for the dimensionless conductance (25) for $\varepsilon=0.5$ :

$$
\begin{aligned}
T_{0.5}^{\mathrm{ana}} & =T_{\Uparrow \Uparrow}+T_{\Downarrow \Downarrow}=\frac{2}{b^{2}} P_{13}^{\prime} P_{13}^{\prime *} \\
& =\frac{8 \delta_{3} \delta_{3}^{*}}{\left(\left|g_{1}+g_{4}\right|^{2}+4 \operatorname{det} g^{*}\right)\left(\left|g_{1}+g_{4}\right|^{2}+4 \operatorname{det} g\right)} .
\end{aligned}
$$

In Eq. (52) we already evaluated part of the numerator that finally yields

$$
\delta_{3} \delta_{3}^{*}=16 \frac{\cos ^{2}\left(\zeta_{+}^{\uparrow}+\zeta_{+}^{\downarrow}\right)}{\cos ^{2}\left(\zeta_{+}^{\uparrow}-\zeta_{+}^{\downarrow}\right)} \sin ^{2} \frac{\Delta n \pi}{2} \cos ^{2} \frac{\left(n_{+}^{\uparrow}+n_{+}^{\downarrow}\right) \pi}{2} .
$$

Furthermore, the denominator of Eq. (55) evaluates to

$$
\begin{aligned}
& 4 \frac{\cos ^{4}\left(\zeta_{+}^{\uparrow}+\zeta_{+}^{\downarrow}\right)}{\cos ^{4}\left(\zeta_{+}^{\uparrow}-\zeta_{+}^{\downarrow}\right)}(1-\cos \Delta n \pi)^{2}+16-16 \frac{\cos ^{2}\left(\zeta_{+}^{\uparrow}+\zeta_{+}^{\downarrow}\right)}{\cos ^{2}\left(\zeta_{+}^{\uparrow}-\zeta_{+}^{\downarrow}\right)} \\
& \quad \times(1-\cos \Delta n \pi) \cos \left(n_{+}^{\uparrow}+n_{+}^{\downarrow}\right) \pi,
\end{aligned}
$$

where we have used the unitarity of $t_{1}, t_{2}\left(\operatorname{det} g \operatorname{det} g^{*}\right.$ $=1)$.

The next step is to average Eqs. (56) and (57) over energy. The result for the averaged dimensionless conductance is conveniently expressed after introducing the mean angle $\bar{\gamma}$ $=\frac{1}{2}\left(\gamma_{+}^{\uparrow}+\gamma_{+}^{\downarrow}\right)$, the mean kinetic-energy quantum number $\bar{n}$ $=\frac{1}{2}\left(n_{+}^{\uparrow}+n_{+}^{\downarrow}\right)$, and the difference angle $\Delta \gamma=\gamma_{+}^{\uparrow}-\gamma_{+}^{\downarrow}$. Then Eq. (14) reads

$$
\cot \bar{\gamma}=\frac{\bar{n}+\frac{1}{2}}{\tilde{\mu} B},
$$

and we obtain the relations

$$
\begin{gathered}
\bar{n}=k_{F} r_{0}-\frac{1}{2}+\sin \frac{\Delta \gamma}{2} \sin \bar{\gamma} \approx k_{F} r_{0}-\frac{1}{2}, \\
\Delta n=\cos \bar{\gamma}+\frac{\tilde{\mu} B}{k_{F} r_{0}} \sin \bar{\gamma}, \\
\tan \Delta \gamma=-\frac{\Delta n}{\tilde{\mu} B+\frac{\bar{n}(\bar{n}-1)}{\tilde{\mu} B}} .
\end{gathered}
$$

Furthermore, we can express $\bar{\gamma}$ and $\Delta n$ in terms of the adiabaticity parameter $Q$ defined in Eq. (4), which elucidates the role of the geometric phase as a measure of adiabaticity,

$$
\cos \bar{\gamma}=\frac{1}{\sqrt{1+Q^{2}}}, \quad \Delta n=\sqrt{1+Q^{2}} .
$$

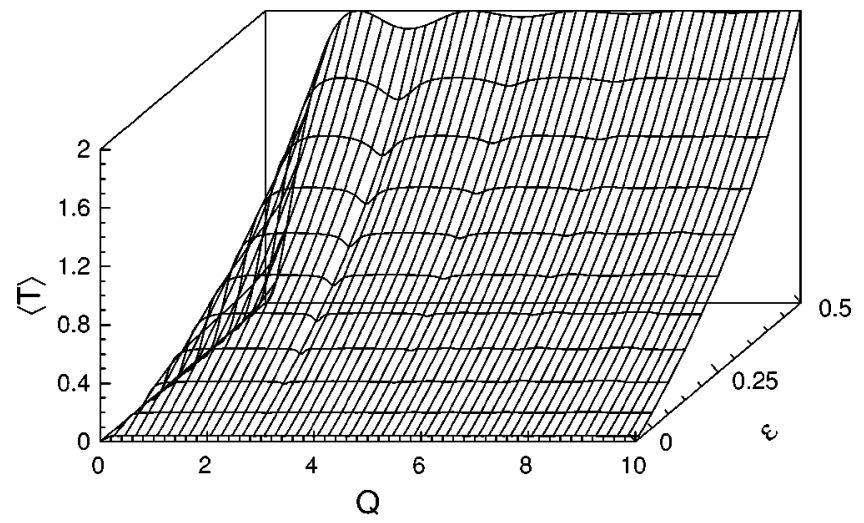

FIG. 9. Same as Fig. 7, but for an Aharonov-Bohm flux $\phi^{\mathrm{AB}}$ $=\phi_{\mathrm{ext}}^{\mathrm{AB}}=\phi_{0} / 2$. For $Q=0$ the conductance is zero. As $Q \rightarrow \infty$, the oscillations diminish and $\langle T\rangle$ depends only on the coupling $\varepsilon$, saturating at $\langle T\rangle=2 \varepsilon /(1-\varepsilon)$. The minima of $\langle T\rangle$ occur at $Q$ $=\sqrt{(2 m+1)^{2}-1}, m \in \mathbb{N}$.

With the last relations we obtain the following approximate result for the averaged dimensionless conductance in the case of maximal coupling strength $(\varepsilon=0.5)$ and no AharonovBohm flux penetrating the ring:

$$
\begin{aligned}
\left\langle T_{0.5}^{\mathrm{ana}}\right\rangle & =\frac{16 \cos ^{2} \bar{\gamma} \sin ^{2} \Delta n \frac{\pi}{2}}{4+\cos ^{4} \bar{\gamma}(1-\cos \Delta n \pi)^{2}} \\
& =\frac{16}{1+Q^{2}} \frac{\sin ^{2}\left(\frac{\pi}{2} \sqrt{1+Q^{2}}\right)}{4+\left(1+Q^{2}\right)^{-2}\left[1-\cos \left(\pi \sqrt{1+Q^{2}}\right)\right]^{2}} .
\end{aligned}
$$

From this equation we immediately recover the zeros of $\left\langle T_{0.5}^{\text {ana }}\right\rangle$ at $\sqrt{1+Q^{2}}=2 m, m \in \mathbb{N}$. In Fig. 8(b), Eq. (59) is compared with the numerical result, pointing out a good agreement of the two curves, with deviations visible only close to the local maxima, where $\langle T\rangle$ is approximated by the Lorentzian prefactor $1 /\left(1+Q^{2}\right)$. The results for $1 \mathrm{D}$ rings presented in Fig. 8 also properly describe the 2D case with one transverse channel. A comparison with the full numerical calculation (presented in paper II of this series) is given in the inset of Fig. 7 and shows the very good agreement between the $2 \mathrm{D}$ results and the $1 \mathrm{D}$ model with $\epsilon=0.316$.

Equation (59) holds in the strong coupling limit $\varepsilon=0.5$. For small coupling strengths, we find that $\langle T\rangle$ is well described by the Lorentzian envelope function

$$
\langle T\rangle_{\mathrm{env}}=2 \frac{\varepsilon}{1-\varepsilon} \frac{1}{1+Q^{2}},
$$

as is demonstrated in Fig. 8(a) for $\varepsilon \leqslant 0.4$. The $\varepsilon$ dependence is identical to the case of spin-independent transport, see Eq. (20). Deviations from this approximation now occur at the positions where $\langle T\rangle$ drops to zero, and these dips become the narrower the smaller $\varepsilon$. 
So far, we investigated the situation where no AharonovBohm flux was penetrating the ring. The result in the presence of a weak homogeneous magnetic field $B_{z}$ corresponding to half a flux quantum is shown in Fig. 9. The averaged dimensionless conductance vanishes in the diabatic limit $Q=0$. This effect is the complement of the asymptotic value $\langle T\rangle \rightarrow 0$ for $Q \rightarrow \infty$ in the case without a magnetic flux, where effectively half a flux quantum can be provided as geometric flux via the geometric phase, cf. Eq. (22). Note, however, that $\phi_{0} / 2$ is the maximal possible geometric flux, achieved in the adiabatic limit, and that geometricphase effects are reduced in nonadiabatic situations (see Fig. 7). In turn, an Aharonov-Bohm flux $\phi^{\mathrm{AB}}=\phi_{0} / 2$ can be compensated by the geometric flux in the adiabatic limit, as is shown in Fig. 9. Furthermore, we now find a $\varepsilon /(1$ $-\varepsilon$ ) dependence of the averaged dimensionless conductance in the adiabatic limit $Q \rightarrow \infty$, which again is complementary to the case $\phi^{\mathrm{AB}}=0$. Also note that for $\phi^{\mathrm{AB}}=\phi_{0} / 2$ local minima occur at the positions $Q=\sqrt{(2 m+1)^{2}-1}$ with $m \in \mathbb{N}$, in contrast to the minima at $Q=\sqrt{4 m^{2}-1}$ for $\phi^{\mathrm{AB}}$ $=0$. However, for $\phi^{\mathrm{AB}}=\phi_{0} / 2$ the dimensionless conductance at the minima is only diminished and does not drop to zero.

\section{Limiting situations: Adiabatic and diabatic regimes}

Finally, we briefly investigate the dimensionless conductance in the adiabatic and diabatic limits for the in-plane magnetic-field geometry. Adiabaticity is characterized by the dominance of the magnetic field over the orbital motion, manifesting itself in

$$
\gamma_{\rho}^{\sigma}=\pi / 2
$$

(cf. the geometric interpretation of the angles $\gamma_{\rho}^{\sigma}$ in Fig. 2). Using $\zeta_{\rho}^{\sigma}=\gamma_{\rho}^{\sigma} / 2$ in Eq. (36) leads to the relation $g_{1}=-g_{4}$ between the matrix elements of the transfer matrices $t_{1}, t_{2}$. When no additional Aharonov-Bohm flux is present, $\phi^{\mathrm{AB}}$ $=0$, we obtain from Eq. (44) $\delta_{3}=0$, yielding via Eq. (45) immediately

$$
T=0
$$

for the dimensionless conductance in the adiabatic limitthis is the result of the destructive interference due to the maximal geometric phase.

For diabatic conditions, the magnetic field does not play any role, and we are in the situation of

$$
\gamma_{\rho}^{\sigma}=0,
$$

as again is clear from the geometric picture. This last equation still holds in the case of a weak-to-moderate homogeneous magnetic field perpendicular to the ring. After straightforward algebra, we recover an equation for the dimensionless conductance that is equivalent to the one derived by Büttiker et al. in Ref. 27 for a 1D ring subject to an external Aharonov-Bohm flux,

$$
T_{\Uparrow \Uparrow}^{\mathrm{diab}}=T_{\Downarrow \Downarrow \downarrow}^{\mathrm{diab}}=\frac{4 \varepsilon^{2}}{1-2 \cos \left(2 \theta_{d}+2 \theta_{\mathrm{AB}}\right)(a+b)^{2}+(a+b)^{4}},
$$

with the dynamic phase $\theta_{d}$ and the Aharonov-Bohm phase $\theta_{\mathrm{AB}}$ introduced in Eq. (19).

\section{SUMMARY}

We have studied spin-dependent electronic transport in 1D rings in the presence of inhomogeneous magnetic fields, in particular for crownlike and wirelike magnetic fields with a varying homogeneous $B_{z}$ component superposed. We find characteristic signatures of geometric phases in the magnetoconductance and in the energy-averaged transmissions.

In the adiabatic situation, the total conductance can be understood as superposition of two independent electron gases $(\| \uparrow\rangle\rangle$ and $\| \downarrow\rangle\rangle)$ that acquire different geometric (Berry) phases due to the different orientation of their spin with or against the magnetic field. This result can be understood within a model of spin-independent transport plus spin-dependent Zeeman-interaction and Berry phase, cf. Figs. 5(a) and 5(d).

In truly nonabiabatic situations, the spins can flip, and the picture is more involved. For the magnetic field originating from a central micromagnet we have identified clear signatures of geometric phases in the calculated magnetoconductance of 1D rings; see Figs. 5(b)-5(d). They appear as interference effects that destroy the regular Aharonov-Bohm oscillations and become increasingly visible towards the adiabatic limit. Recent experiments ${ }^{22}$ were still performed under rather diabatic conditions that are not favorable for the observation of geometric phases. A generalization of the analytical approach presented here to disorder effects and to diffusive one-dimensional rings appears as promising direction.

For the special case of an in-plane magnetic field we investigated the spin-flip effect of Refs. 1 and 24, which originally was uncovered in numerical simulations of $2 \mathrm{D}$ rings. The 1D model allows for a rigorous analytical explanation and proof of this quantum interference effect that does not depend on the degree of adiabaticity. For polarized incident electrons, a small external Aharonov-Bohm flux can be used to control spin flips and to tune the polarization of transmitted electrons. This spin-flip effect represents a promising control tool in future spintronics engineering, since it also prevails for rings with Rashba spin-orbit coupling, ${ }^{32}$ where the intrinsic effective magnetic field takes the role of the applied inhomogeneous field in the present context.

\section{ACKNOWLEDGMENTS}

We thank J. Biberger, J. Fabian, and D. Weiss for useful discussions. M.H. gratefully acknowledges financial support from the Alexander von Humboldt Foundation. 
${ }^{1}$ D. Frustaglia, M. Hentschel, and K. Richter, following paper, Phys. Rev. B 69, 155327 (2004).

${ }^{2}$ Y. Aharonov and D. Bohm, Phys. Rev. 115, 485 (1959).

${ }^{3}$ For an early comprehensive review see, e.g., S. Washburn and R.A. Webb, Rep. Prog. Phys. 55, 1311 (1992).

${ }^{4}$ G.A. Prinz, Science 282, 1660 (1998); J. M Kikkawa and D.D. Awschalom, Nature (London) 397, 139 (1999); S.A. Wolf, D.D. Awschalom, R.A. Buhrman, J.M. Daughton, S. von Molnár, M.L. Roukes, A.Y. Chtchelkanova, and D.M. Trege, Science 294, 1488 (2001).

${ }^{5}$ M.V. Berry, Proc. R. Soc. London, Ser. A 392, 45 (1984).

${ }^{6}$ Y. Aharonov and J. Anandan, Phys. Rev. Lett. 58, 1593 (1987).

${ }^{7}$ Geometric Phases in Physics, edited by A. Shapere and F. Wilczek (World Scientific, Singapore, 1989).

${ }^{8}$ A. Yacoby, M. Heiblum, D. Mahalu, and H. Shtrikman, Phys. Rev. Lett. 74, 4047 (1995).

${ }^{9}$ R. Schuster, E. Buks, M. Heiblum, D. Mahalu, V. Umansky, and H. Shtrikman, Nature (London) 385, 417 (1997).

${ }^{10}$ W.G. van der Wiel, S. De Franceschi, T. Fujisawa, J.M. Elzerman, S. Tarucha, and L.P. Kouwenhoven, Science 289, 2105 (2000).

${ }^{11}$ Y. Ji, M. Heiblum, D. Sprinzak, D. Mahalu, and H. Shtrikman, Science 290, 779 (2000).

${ }^{12}$ Y. Ji, M. Heiblum, and H. Shtrikman, Phys. Rev. Lett. 88, 076601 (2002).

${ }^{13}$ J. Göres, D. Goldhaber-Gordon, S. Heemeyer, M.A. Kastner, H. Shtrikman, D. Mahalu, and U. Meirav, Phys. Rev. B 62, 2188 (2000)

${ }^{14}$ K. Kobayashi, H. Aikawa, S. Katsumoto, and Y. Iye, Phys. Rev. Lett. 88, 256806 (2002).

${ }^{15}$ D. Loss, P. Goldbart, and A.V. Balatsky, Phys. Rev. Lett. 65, 1655 (1990).

${ }^{16}$ A. Stern, Phys. Rev. Lett. 68, 1022 (1992).

${ }^{17}$ A. Stern, in Mesoscopic Electron Transport, edited by L.L. Sohn,
L.P. Kouwenhoven, and G. Schön (Kluwer Academic, New York, 1997).

${ }^{18}$ A.G. Aronov and Y.B. Lyanda-Geller, Phys. Rev. Lett. 70, 343 (1993).

${ }^{19}$ Y. Lyanda-Geller, I.L. Aleiner, and P.M. Goldbart, Phys. Rev. Lett. 81, 3215 (1998).

${ }^{20}$ D. Frustaglia and K. Richter, Found. Phys. 31, 399 (2001).

${ }^{21}$ M.J. Yang, C.H. Yang, K.A. Cheng, and Y.B. Lyanda-Geller, cond-mat/0208260 (unpublished).

${ }^{22}$ P. D. Ye, S. Tarucha, and D. Weiss, Proceedings of the EP2DS, Jerusalem (1998).

${ }^{23}$ J.A. Katine, F.J. Albert, R.A. Buhrman, E.B. Myers, and D.C. Ralph, Phys. Rev. Lett. 84, 3149 (2000).

${ }^{24}$ D. Frustaglia, M. Hentschel, and K. Richter, Phys. Rev. Lett. 87, 256602 (2001).

${ }^{25}$ E.I. Rashba, Fiz. Tverd. Tela (Leningrad) 2, 1224 (1960) [Sov. Phys. Solid State 2, 1109 (1960)]; Y.A. Bychkov and E.I. Rashba, J. Phys. C 17, 6039 (1984).

${ }^{26}$ Y.S. Yi, T.Z. Qian, and Z.B. Su, Phys. Rev. B 55, 10631 (1997).

${ }^{27}$ M. Büttiker, Y. Imry, and M.Ya. Azbel, Phys. Rev. A 30, 1982 (1984).

${ }^{28}$ M. Hentschel, Dissertation, Technische Universität Dresden (2001).

${ }^{29}$ A. Nogaret, S.J. Bending, and M. Henini, Phys. Rev. Lett. 84, 2231 (2000).

${ }^{30}$ J.-B. Yau, E.P. De Poortere, and M. Shayegan, Phys. Rev. Lett. 88, 146801 (2002).

${ }^{31}$ Note that $|\uparrow\rangle$ always means a spin in direction of the magnetic field. For a circular magnetic field, consequently, the spin of a $|\uparrow\rangle$ electron points into opposite directions at the entrance and the exit of the ring, respectively. This convention is opposite to the one in Refs. 1 and 24 but more appropriate for the nonplanar magnetic fields discussed here.

${ }^{32}$ D. Frustaglia and K. Richter, cond-mat/0309228 (unpublished). 